\title{
Deep MIPS Observations of the IC 348 Nebula: Constraints on the Evolutionary State of Anemic Circumstellar Disks and the Primordial-to-Debris Disk Transition
}

\author{
Thayne Currie ${ }^{1}$, Scott J. Kenyon ${ }^{1}$ \\ tcurrie@cfa.harvard.edu, skenyon@cfa.harvard.edu
}

\begin{abstract}
We describe new, deep MIPS photometry and new high signal-to-noise optical spectroscopy of the 2.5 Myr-old IC 348 Nebula. To probe the properties of the IC 348 disk population, we combine these data with previous optical/infrared photometry and spectroscopy to identify stars with gas accretion, to examine their mid-IR colors, and to model their spectral energy distributions. IC 348 contains many sources in different evolutionary states, including protostars and stars surrounded by primordial disks, two kinds of transitional disks, and debris disks. Most disks surrounding eary/intermediate spectral-type stars $\left(>1.4 \mathrm{M}_{\odot}\right.$ at 2.5 Myr) are debris disks; most disks surrounding solar and subsolar-mass stars are primordial disks. At the 1-2 $\sigma$ level, more massive stars also have a smaller frequency of gas accretion and smaller mid-IR luminosities than lower-mass stars. These trends are suggestive of a stellar mass-dependent evolution of disks, where most disks around high/intermediate-mass stars shed their primordial disks on rapid, 2.5 Myr timescales. The frequency of MIPS-detected transitional disks is $\approx 15-35 \%$ for stars plausibly more massive than $0.5 \mathrm{M}_{\odot}$. The relative frequency of transitional disks in IC 348 compared to that for 1 Myr-old Taurus and 5 Myr-old NGC 2362 is consistent with a transition timescale that is a significant fraction of the total primordial disk lifetime.
\end{abstract}

Subject headings: stars: pre-main-sequence-planetary systems: formation planetary systems: protoplanetary disks

\section{Introduction}

Most $\leq 1$ Myr-old stars are surrounded by optically-thick, accreting primordial disks, which produce strong near-to-mid infrared (IR) emission $\left(\mathrm{L}_{d} / \mathrm{L}_{\star} \geq 0.1\right.$; Kenyon and Hartmann

\footnotetext{
${ }^{1}$ Harvard-Smithsonian Center for Astrophysics, 60 Garden St. Cambridge, MA 02140
} 
1995). The gas and dust in these disks comprise the building blocks of planets. As stars age, the dust grains grow, settle toward the midplane, and become incorporated into planetesimals. Circumstellar gas depletes by accretion onto the star (Hartmann et al. 1998). All of these processes reduce the amount and frequency of disk emission on timescales of $\sim 3-7$ Myr for most stars (Hernandez et al. 2007; Hillenbrand 2008; Currie et al. 2009).

By $\sim 10$ Myr, most stars do not have optically-thick primordial disks (Currie et al. 2007a; Hillenbrand 2008). Disks around these older stars are typically debris disks (Currie et al. 2008a), which have weaker, optically-thin dust emission $\left(\mathrm{L}_{d} / \mathrm{L}_{\star} \lesssim 10^{-3}\right)$ and lack evidence for circumstellar gas accretion. Because stellar radiation - radiation pressure and PoyntingRobertson drag - or stellar wind drag can remove dust in debris disks on timescales much less than the age of the star, their dust requires a replenishment source, which is supplied by active icy or rocky planet formation (e.g. Backman and Paresce 1993; Kenyon and Bromley 2004, 2008). Because debris disks lack copious amounts of circumstellar gas needed to form gas giant planets, identifying when most primordial disks turn into debris disks pinpoints an empirical upper limit for the formation timescale for gas giant planets.

Recent Spitzer Space Telescope studies of 1-5 Myr-old clusters indicate that primordial disks disappear faster around early type, high/intermediate-mass stars than they do around late type, low-mass stars (Carpenter et al. 2006; Lada et al. 2006; Hernandez et al. 2007; Currie et al. 2009). By 5 Myr, debris disks completely dominate the disk population around high/intermediate-mass stars. At $5 \mathrm{Myr}$, most disks around lower-mass stars appear to have inner holes and/or extremely low warm dust masses (Dahm and Carpenter 2009; Currie et al. 2009), which indicates that they may be disappearing and actively making the primordialto-debris disk transition. Thus, the epoch at which most high/intermediate-mass stars make the primordial-to-debris disk transition must occur at an age prior to $5 \mathrm{Myr}$ and may be identified by mid-IR observations of younger clusters.

With a median age of 2-3 Myr, stars in the IC 348 Nebula (Herbig 1998) may help to constrain the epoch when most primordial disks around high/intermediate-mass stars turn into debris disks. Cluster membership is well determined (e.g. Herbig 1998; Luhman et al. 1998, 2003; Muench et al. 2007). At only 320 pc distant, the cluster provides a sensitive probe of low-levels of disk emission in spite of its high infrared background.

Recently, Lada et al. (2006) and Muzerolle et al. (2006) analyzed Spitzer Cycle 1 IRAC and MIPS IC 348 data and found evidence for substantial disk evolution. Based on the IRAC $3.6 \mu m$ to $8 \mu m$ flux slope, Lada et al. (2006) show that only $\approx 30 \%$ of cluster stars have strong ('thick') IRAC excess emission comparable to stars with primordial disks (e.g. most disk-bearing stars in Taurus). Another $\approx 23 \%$ have weaker ('anemic') IRAC excess emission, and $\approx 44 \%$ lack IRAC excess emission ('diskless'). However, inferring the 
physical state of disks from the observed IRAC slope is not straightforward. Both debris disks and 'evolved' primordial disks ('transitional' disks: those with lower disk masses and/or inner holes) can have weak IRAC-excess emission (Currie et al. 2007b, 2009). Furthermore, while SED analysis including longer wavelength data (e.g. $24 \mu \mathrm{m}$ ) helps to break this degeneracy (Currie et al. 2009), only $\approx 30 \%$ of IC 348 stars have Cycle 1 MIPS detections. Therefore, deeper MIPS data coupled with other diagnostics of the disk evolutionary state (e.g. gas accretion signatures) would help to make IC 348 a better laboratory for studying disk evolution.

In this paper, we combine new, high signal-to-noise optical spectra and new, deeper MIPS $24 \mu \mathrm{m}$ and $70 \mu \mathrm{m}$ photometry of IC 348 with archival data to study disk evolution in more detail. We first use the optical spectra to search for evidence of circumstellar gas accretion, which is absent in debris disks. Deeper MIPS data is then used to establish a much larger sample of disk-bearing stars. From these data, we compare the disks SEDs to models to constrain their evolutionary states, to compare disk properties around highmass stars to low-mass stars, and to investigate the timescale for and the morphology of the primordial-to-debris disk transition.

\section{New Observations and Archival Data}

\subsection{New Optical Spectroscopy of High/Intermediate-Mass IC 348 Stars}

To investigate accretion signatures for IC 348 stars, we took high signal-to-noise spectra of cluster stars with published spectral types earlier than K0. Our primary motivation for selecting this sample is that these stars lack recent high signal-to-noise observations. High signal-to-noise observations are effective in identifying gas accretion signatures around solar and subsolar-mass cluster stars not previously known as accretors (Dahm 2008). New observations of early/intermediate type stars may identify sources with emission line reversal in the cores of their $\mathrm{H}_{\alpha}$ absorption lines, a feature which is consistent with gas accretion (Dahm 2008).

Selected IC 348 stars were observed on March 1, 2008 with the FAST spectrograph on the 1.5m Tillinghast telescope at Fred Lawrence Whipple Observatory (Fabricant et al. 1998). The spectra were taken using a $300 \mathrm{~g} \mathrm{~mm}^{-1}$ grating blazed at $4750 \AA$ and a 3 " slit. These spectra cover $3700-7500 \AA$ at $6 \AA$ resolution. The data were processed using the standard FAST reduction pipeline (Fabricant et al. 1998). The continuum signal to noise at $\approx 6550$ $\AA$ ranged from 80 to 170 . To derive line fluxes in $\mathrm{H}_{\alpha}$, we fit the line to a gaussian profile with standard IRAF routines. The properties of each source and their spectral features are 
listed in Table 1 .

\subsection{Archival Optical/Near-IR Ground-based Data and Archival Spitzer 3.6-24 $\mu \mathrm{m}$ Data}

We collected published optical and infrared photometric data and spectroscopic data for IC 348 stars from the Lada et al. (2006) catalog. This catalog yields photometric data from $3.6 \mu \mathrm{m}$ through $24 \mu \mathrm{m}$, optical extinction $\left(\mathrm{A}_{V}\right)$, spectral types, and IRAC SED slopes (thick, anemic or diskless). We added BVI photometry of IC 348 sources from Luhman et al. (2003), Herbig (1998), and Luhman et al. (2005), near-IR JHKs photometry from 2MASS, and bolometric luminosities from Muench et al. (2007).

Nearly all of the 307 sources in the Lada et al. (2006) sample have spectral types (304) and $\mathrm{A}_{V}$ measurements (295). Most have 2MASS data (280), I-band data (270), and IRAC $8 \mu m$ data (265). Completeness in the MIPS $24 \mu m$ band is poorer (88 sources). Many sources without MIPS detections are either not detected in the $8 \mu \mathrm{m}$ IRAC channel (35) and/or have photospheric colors through $8 \mu m$ (128).

\subsection{New MIPS $24 \mu m$ and $70 \mu m$ Photometry}

IC 348 was reobserved in five separate epochs with the Multiband Imaging Photometer for Spitzer (MIPS) on September 23-27, 2007 (Observing Keys 22961920, 22962176, 22962432, 22962688, and 22962944; PI J. Muzerolle). The area of coverage is $\approx 0.5$ square degrees. A series of papers focused on analyzing the IC 348 sources with variable mid-IR emission will be published later using these MIPS data and IRS spectra (J. Muzerolle, K. Flaherty, et al. 2009a,b, in prep.). Our motivation for using these data is to identify new 24 $\mu m$ detections of cluster stars from stacking together pointings from the five epochs. Preliminary findings indicate that some low-mass IC 348 stars have $24 \mu \mathrm{m}$ emission varying by up to several tenths of magnitudes from epoch to epoch (J. Muzerolle, pvt. comm.). Therefore, by combining observations from these epochs together, we sacrifice any information about disk variability. The resulting photometry is an epoch-averaged value: photometry for low-mass stars from individual epochs may differ by several tenths of magnitudes. 


\subsubsection{MIPS 24 4 m Image Processing, Photometry, and Source Matching}

Using MOPEX (Makovoz and Marleau 2005), we first mosaic the Basic Calibration Data (BCD data) together using a bicubic spline interpolation. In constructing the final mosaic, we rejected and reinterpolated over pixels with values more than $5 \sigma$ away from the (clipped) mean value. Standard deviation mosaics were also constructed. The Basic Calibration Data (BCD) frames have an integration time of $3.67 \mathrm{~s}$; most pixels had $\gtrsim 110$ separate pointings. The typical cumulative integration time/pixel is then $\approx 410 \mathrm{~s}$, about a factor of five greater than the Cycle 1 data analyzed in Lada et al. (2006).

Source detection and photometry was performed using APEX (Makovoz and Khan 2005) in single-frame mode. In a first pass, we identified sources as clusters of 4-50 pixels in the signal-to-noise mosaic, which is produced by dividing the background-subtracted science mosaic by a map of the background fluctuations (produced from the gaussnoise module from APEX). We used the 'peak' algorithm with a 45x45 pixel box to define the background and a $2.5 \sigma$ detection threshold. While these settings yielded robust detections of many faint stars, they sometimes 'deblended' single stars, especially those with bright Airy rings. Furthermore, these settings missed detecting some sources located in regions with smaller-scale fluctuations in the background (e.g. near the center of the nebula). Therefore, in a second pass, we chose a $25 \times 25$ pixel box, using a $5 \sigma$ detection threshold and the 'combo' algorithm, which alleviated these problems.

Pixel Response Function (PRF) photometry was performed on detected sources, using an empirical PRF derived from bright $(\mathrm{S} / \mathrm{N}>50-100)$ stars in low, uniform background regions resampled from the native pixel scale $(\sim 2.45$ " / pixels $)$ by a factor of four. The empirical PRF and model PRF supplied by the Spitzer Science Center show strong agreement. PRF fitting was performed over a $5 \times 5$ pixel area with fitting tolerance of $10^{-3}$. Photometric errors were estimated from both the signal-to-noise from background fluctuations (due to both photon noise and nebulosity) and errors in the PRF fitting. We detect $\approx 5,900$ candidate point sources; the source counts peak at $[24] \sim 11$.

To understand the empirical photometric scatter in our MIPS photometry and to identify candidate pre-main sequence stars, we cross-correlated our MIPS-24 $\mu m$ catalog with the 2MASS point source catalog. Using a 4" matching radius, we found 451 matches. The left panel of Figure 2 shows the $\mathrm{J}-\mathrm{K}_{s}$ vs. $\mathrm{K}_{s^{-}}[24]$ colors for these sources. Overplotted as a dotted line is the locus of photospheric colors from the Kurucz-Lejeune stellar atmosphere models (Kurucz 1993; Lejeuene et al. 1997) as listed by the STAR-PET tool available on the Spitzer Science Center website. Sources with photometric uncertainties in their $\mathrm{K}_{s}-[24]$ colors $<0.2$ magnitudes (grey dots) either lie well redward of the locus (IR excess sources) or are tightly clustered along the locus. Sources with larger photometric uncertainties (black 
dots) appear to have a wider dispersion in color.

Figure 2 (right panel) shows the [24] vs. $\mathrm{K}_{s^{-}}[24]$ color magnitude diagram of these sources. The vertical curves (dotted, dashed, and three-dash dots lines) show the median 1 and $2 \sigma$ uncertainties in $\mathrm{K}_{s^{-}}[24]$ colors computed in 1-magnitude bins, assuming an intrinsic dispersion of 0.1 magnitudes centered on $\mathrm{K}_{s^{-}}[24]=0.25$. There is little blueward deflection of colors outside of the $1 \sigma$ limit; the $2 \sigma$ limit completely contains all sources with colors bluer than $\mathrm{K}_{s^{-}}[24]=0.25$. We do not find a significant trend between photometric scatter and background level (not shown). Thus, if there is an intrinsic $\gtrsim 0.1$ magnitude dispersion in colors due to differences in spectral type/reddening, the observed photometric scatter is explainable in terms of the computed photometric uncertainties for MIPS and 2MASS.

Using a matching radius of 4", we identify 27 IC 348 cluster stars with new $24 \mu \mathrm{m}$ detections. These detections were confirmed by inspection of the final mosaic image, the background-subtracted mosaic, and the signal-to-noise mosaic. The coordinates were then checked against the positions of other sources to identify possible blends. One source, ID 9099, is 3" from ID 99. Its catalog position is offset from the MIPS detection by 2.83". Another source, ID 1937, is within 4.3" of ID 1928 and has an offset of 3" between its catalog position and position on the MIPS-24 $\mu \mathrm{m}$ mosaic. We remove these sources from the list, leaving 25 non-blended sources detected using APEX.

To find missed sources, we manually searched the positions of the remaining 192 stars in the Lada et al. (2006) catalog without APEX detections. This search yielded two additional detections (IDs 124 and 373). PSF-fitting photometry was performed on these two sources using the IDL version of the DAOPHOT package with empirical PSFs constructed from bright stars uncontaminated by nebulosity $(\mathrm{S} / \mathrm{N}>50-100)$. With these new data added, the Lada et al. (2006) catalog includes 27 sources with new detections, bringing the total number of MIPS-detected stars to 115 . This new sample is a $\sim 30 \%$ larger than the sample analyzed in Lada et al. (2006). Table 2 lists the properties of the IC 348 stars with new MIPS $24 \mu m$ detections.

\subsubsection{MIPS $70 \mu \mathrm{m}$ Image Processing, Photometry, and Source Matching}

Using MOPEX, we mosaiced both the MIPS-70 $\mu \mathrm{m}$ BCD data and the filtered BCD data. As with the $24 \mu \mathrm{m}$ data, we combined the individual frames together, rejecting and reinterpolating over pixels more than $5 \sigma$ from the (clipped) mean pixel value. We constructed the final mosaic images and standard deviation mosaics. The MIPS $70 \mu m$ data have an intergration time/pixel of 4.19 s for the BCD frames and a cumulative integration time of $\approx$ 
210s, a factor of five improvement over previous data presented in Muench et al. (2007).

Source identification and photometry were performed using APEX in single-frame mode. For source detection on both the unfiltered and filtered mosaics, we chose a $5 \sigma$ detection threshold for initial cluster detection, limiting image segmentation to candidate sources between 5 and 250 pixels in area. Because there were too few bright point sources detected at $70 \mu \mathrm{m}$, we used the model PRF for photometry instead of constructing an empirical one. Each source was fit by the model PRF over a $7 x 7$ pixel area with a resampling factor of four and a fitting tolerance of $10^{-3}$. Photometric errors were calculated in the same manner as the $24 \mu \mathrm{m}$ data. Because sources brighter than $\approx 1$ Jy can have their fluxes attenuated on the filtered mosaic, we chose the unfiltered mosaic for $70 \mu \mathrm{m}$ photometry.

Because MIPS-70 $\mu \mathrm{m}$ is not sensitive to stellar photospheric fluxes of stars at the distance of IC 348, we cannot investigate the photometric reliability of the $70 \mu \mathrm{m}$ as we did with the $24 \mu \mathrm{m}$ data. We matched the APEX $70 \mu \mathrm{m}$ source list to the Lada et al. (2006) catalog using a 5" matching radius. We find 6 sources in the MIPS-70 catalog with matches in the Lada et al. (2006) catalog, four of which (IDs 31, 67, 10343, and 10352) are new detections. We visually confirmed these detections on both the unfiltered and filtered mosaics. Table 3 lists the properties of stars detected at MIPS $70 \mu \mathrm{m}$.

\subsubsection{MIPS Upper Limits for Unmatched Sources}

Figure 3 shows a RGB mosaic image produced from our $24 \mu \mathrm{m}$ and $70 \mu \mathrm{m}$ mosaics and the post-BCD mosaic of the IRAC $8 \mu \mathrm{m}$ data used by Lada et al. (2006). Most of the IC 348 sources in the Lada et al. (2006) catalog are located within regions of enhanced nebulosity. While nebulosity does not serious impede detections of most of the Lada et al. (2006) sources at $8 \mu \mathrm{m}$, the decreased sensitivity of MIPS and enhanced nebular emission at $24 \mu \mathrm{m}$ and 70 $\mu m$ greatly increase the number of nondetections at these wavelengths.

To compute the $5 \sigma$ upper limits for sources lacking detections, we use the $24 \mu \mathrm{m}$ and 70 $\mu m$ uncertainty images as maps of the noise. The median $5 \sigma$ flux density is computed over

a $3 \times 3$ region centered on the coordinates of each Lada et al. (2006) source. To calculate the $5 \sigma$ flux upper limit for MIPS $24 \mu \mathrm{m}$, we multiply the flux density by a pixel area of $\pi(2.45$ pixels $)^{2}$ and use an aperture correction of 1.697 . For the $70 \mu \mathrm{m}$ data, we multiply the flux density by a pixel area of $\pi(1.62 \text { pixels })^{2}$ and use an aperture correction of 1.735 . Upper limits at $24 \mu \mathrm{m}$ varied from $\approx 0.3 \mathrm{mJy}([24] \sim 11.0)$ in low (black) background regions to $\approx 180$ mJy $([24] \sim 4)$ in the high background regions of the nebula center (reddish-white/white). Typical upper limits at $70 \mu \mathrm{m}$ varied from $\approx 30 \mathrm{mJy}$ in low background regions to $\approx 420$ 
mJy in the center of the nebula.

\subsubsection{Comparisons with Previous IC 348 MIPS Photometry}

In Figure 4, we compare our MIPS photometry with results from Lada et al. (2006) and Muench et al. (2007) derived from Cycle 1 data. The left panel contrasts our [24] magnitudes and upper limits, where grey circles represent magnitudes for IC 348 members detected from both data sets and crosses represent $2 \sigma$ upper limits for IC 348 members lacking detections from both data sets. Compared to the Lada et al. (2006) photometry, our [24] photometry exhibits a systematic offset of -0.0075 magnitudes and a median difference of 0.05 magnitudes.

While our $70 \mu \mathrm{m}$ flux for ID-13 is just over half that quoted by Muench et al. (2007), our measurement for ID-51 is nearly identical to that from Muench et al. (2007).

In the cases where the [24] photometry differs by more than $0.2-0.3$ magnitudes, there is evidence that our photometry is more accurate. For example, ID-7 has $[24]=7.61$ from this work and $[24]=6.95$ from Lada et al. (2006). Comparing its flux densities on our mosaic and the Cycle 1 data post-BCD mosaic indicates that variability is unlikely to explain the strong disagreement in photometry. Lada et al. (2006) find that ID-7 is about equal in brightness to nearby ID-8. However, it is clear that ID-7 is much fainter because its peak flux density above the local background is just over half that of ID-8's $\left(\approx 3.7 \mathrm{MJy} \mathrm{sr}^{-1}\right.$ vs. $\left.7.1 \mathrm{MJy} \mathrm{sr}^{-1}\right)$. Thus, ID-7 should be $\approx 0.7$ magnitudes fainter than ID-8, as confirmed by our measurements $\left([24]_{I D-7}=7.61\right.$ and $\left.[24]_{I D-8}=6.89\right)$.

ID-19 is another source whose discrepant photometry probably cannot be due to variability $([24]=6.24$ here, $[24]=4.6$ from Lada et al. 2006). This source is located near the center of the nebula, where the background flux density increases from $\approx 75 \mathrm{MJy} \mathrm{sr}{ }^{-1}$ to $\approx$ $98 \mathrm{MJy} \mathrm{sr}^{-1}$ over $\approx 20$ ". Unless we assume that the average background is $\approx 75 \mathrm{MJy} \mathrm{sr}^{-1}$, we fail to reproduce the Lada et al. (2006) result from either PRF fitting or PSF fitting. However, there is a significant slope in the local background at constant declination near ID-19; thus, the average or median background is much higher than $75 \mathrm{Myr} \mathrm{sr}^{-1}$. Independent MIPS $24 \mu \mathrm{m}$ photometry yields values for ID-7 and ID-19 that are similar to ours (J. Hernandez 2009, private communication).

The left panel of Figure 4 also compares our upper limits for nondetections (crosses). Assuming the Lada et al. (2006) upper limits are accurate, The dotted line corresponds to the predicted locus of upper limits given our integration time in the photon noise limit $\left(2.5 \log _{10}(\sqrt{5}) \sim 0.87\right)$. Because nebular emission dominates the background in most regions, our upper limits should lie to the left of the line. However, most of our upper limits are 
clearly fainter than predictions based on Lada et al.'s upper limits. Independent analyses also find significantly fainter upper limits (J. Hernandez 2009, private communication). The disagreement likely arises because Lada et al. (2006) add together the background flux and its uncertainty, which would determine the upper limit of the source plus background, not the upper limit of the source flux, since the background is an astronomically distinct object.

Even though the new MIPS data are deeper, the right panel of Figure 4 shows that our photometric uncertainties are larger than those listed by Lada et al. (2006). Our results agree with those of Lada et al. much better if we assume that the background uncertainty (from nebulousity) does not contribute to photometric uncertainty. In spite of some disagreements with Lada et al. (2006)'s photometry, results of the analysis presented in the proceeding sections are not qualitatively affected by which photometric catalog is used for previously detected stars.

\section{Results from New Data}

We now use new data to expand on Lada et al's investigations of the IC 348 disk population. First, we investigate accretion signatures to probe the presence or absence of warm circumstellar gas in disks surrounding cluster stars. We then analyze the mid-IR colors of stars with new MIPS detections to identify IR excess sources. We then follow the analysis methods of Currie et al. (2009) to model the SEDs of new sources to constrain their evolutionary states and to refine the Lada et al. (2006) analysis of previously detected sources.

\subsection{Gas Accretion Signatures and Upper Limits}

To investigate the gas content of high/intermediate-mass stars, we consider line fluxes derived from new, higher signal-to-noise optical spectra. Our spectra show a variety of $\mathrm{H}_{\alpha}$ morphologies (Figure 11). IDs 3, 8, and 30 lack $2 \sigma$ fluctuations that could be interpreted as $\mathrm{H}_{\alpha}$ emission in the central absorption core. The spectra of ID-2 shows a flattening of the $\mathrm{H}_{\alpha}$ absorption core, though it lacks unambiguous evidence for emission line reversal. Higher-resolution spectroscopy suggests that this source may have emission line reversal (S. Dahm, unpublished) as noted in Dahm (2008).

ID-6 has $\mathrm{H}_{\alpha}$ in absorption but a smaller measured equivalent width than the typical width for early/mid G stars $(\sim 3-4 \AA)$. Thus, the $\mathrm{H}_{\alpha}$ line may be filled in by emission. However, the spectrum shows no clear evidence for an emission line core. Furthermore, 
ID-6 is very chromospherically active: it has the largest X-ray luminosity of any Chandradetected source in IC 348, $\mathrm{L}_{x} \sim 1.96 \times 10^{31} \mathrm{ergs} \mathrm{s}^{-1}$ (Preibisch and Zinnecker 2001, 2002). Such activity should produce weak $\mathrm{H}_{\alpha}$ emission and result in a weakened absorption line.

ID-21 shows $\mathrm{H}_{\alpha}$ in emission with $\mathrm{EW}\left(\mathrm{H}_{\alpha}\right) \sim 5 \AA$ consistent with previous results. Surprisingly, ID-19, an A2 star not previously known to be accreting, also shows evidence for $\mathrm{H}_{\alpha}$ emission consistent with accretion. Even though the line is in absorption, a central emission core is clearly visible.

For the sources lacking clear evidence for accretion, we can rule out $\mathrm{H}_{\alpha}$ line emission hiding in the noise at a level observed in late-type stars. We estimate the upper limit of the $\mathrm{H}_{\alpha}$ flux following Allen (1995). First, from the spectral type and extinction, we derive the expected dereddened continuum flux in the Cousins $\mathrm{R}$ band. We then measure the equivalent width of the continuum noise near the $\mathrm{H}_{\alpha}$ line. An upper limit to the line flux then results: $\operatorname{Flux}_{H_{\alpha}, u l} \approx \mathrm{EW}($ noise $) \times \mathrm{R}_{\text {flux }}$.

Finally, we derive an upper limit to the accretion rate scaled to that of ID-21 from $\mathrm{L}_{H_{n}}$ $\propto \mathrm{G}^{M} \mathrm{M}_{\star} / \mathrm{R}_{\star}$. We determine $\mathrm{R}_{\star}$ from the derived values for $\mathrm{T}_{e}$ and $\mathrm{L}_{\star}$ from Muench et al. (2007) and use the D'Antona and Mazzitelli (1994) isochrones to estimate stellar masses. Although the theoretical ages of young stars are uncertain (e.g., D'Antona and Mazzitelli 1994; Siess et al. 2000; Baraffe et al. 1998) the masses derived from $\mathrm{L}_{\star}$ and $\mathrm{T}_{e}$ using different isochrones are generally consistent. As shown in Table 1 , the $\mathrm{H}_{\alpha}$ line flux (accretion rate) for sources lacking evidence for gas accretion is $\lesssim 10 \%(\lesssim 0.5-10 \%)$ of the $\mathrm{H}_{\alpha}$ emission (accretion rate) in ID-21. These upper limits also rule out accretion at a rate equal to that for ID-19.

As with any pre-main sequence star, it is still possible that the stars lacking clear accretion signatures are accreting at undetectable rates. Furthermore, it is possible that disks surrounding these stars lack warm circumstellar gas accreting onto the star but contain some gas in outer disk regions. Their optical spectra and resulting upper limits on accretion are more consistent with a lack of accretion and thus a heavily depleted or even nonexistent gas reservoir.

\subsection{Mid-to-Far IR Colors of IC 348 Stars with new MIPS Detections}

\subsubsection{MIPS 24 $\mathrm{mm}$ Colors}

Figure 5 shows the [3.6]-[8] vs. [8]-[24] color-color diagram for new MIPS detections. Overplotted are the $1 \sigma$ error bars in each filter. We include a reddening vector of length 
$\mathrm{A}_{V}=20$ (the maximum extinction), using the extinction laws from Indebetouw et al. (2005) and Mathis (1990). Most newly-detected stars have spectral types later than K0 (left panel; grey dots); only one new detection has an earlier spectral type (left panel; black dot).

Cluster stars with new $24 \mu \mathrm{m}$ detections generally have excess emission consistent with reprocessed radiation from circumstellar disks. Most new detections have an observed [3.6][24] color redder than $\sim 1.5-2$ magnitudes. In contrast, [3.6]-[24] $\leqslant 0.5$ for bare stellar photospheres for stars of most spectral types (e.g. Currie. Plavchan, and Kenyon 2008). Because the maximum extinction for cluster members of $A_{V} \sim 20$ (shown as an arrow) reddens the stars by less than $\sim 0.5$ magnitudes, high extinction is unlikely to explain these colors. Therefore, the excess emission plausibly arises from circumstellar material.

The distribution of mid-IR colors is consistent with results for previously detected cluster stars analyzed in Lada et al. (2006). Most stars identified as 'diskless' (right panel; light grey dots) by Lada et al. (2006) either have photospheric MIPS emission or excess emission less than 2 magnitudes. Their MIPS excess emission is less than that from stars with strong ('thick') IRAC excess (right panel; black dots) and stars with weak ('anemic') excess (right panel; dark grey dots). Both 'anemic' and 'thick' disks have a range of [8]-[24] colors from $\sim 2$ to 4.5 and differ primarily by their levels of IRAC emission.

To compare the mid-IR colors of IC 348 sources with fiducial values, we overplot colors of the median Taurus SED (asterisk) reddened to $\mathrm{E}(\mathrm{B}-\mathrm{V}) \sim 1.56$, the mean value for IC 348 stars. To produce the Taurus colors, we use fluxes from Furlan et al. (2006) for the $8 \mu \mathrm{m}$ and $24 \mu \mathrm{m}$ filters and approximate the $3.6 \mu \mathrm{m}$ flux from D'Alessio et al. (1999). The Taurus colors lie within the distribution of colors for 'thick' sources in IC 348.

While many newly-detected stars have levels of excess emission typical of primordial disks, the full range of IRAC vs. MIPS colors hints at a diversity in disk properties. Two stars have nearly zero [3.6]-[8] color and [8]-[24] $\gtrsim 3$. These colors are consistent with stars whose disks have inner holes cleared of dust and are optically-thick at larger stellocentric distances. A second group of stars also have zero IRAC color but may have weak $24 \mu \mathrm{m}$ excess emission ([8]-[24] 0.5-2). These colors are consistent with the colors of either debris disks or remnant protoplanetary disks that are actively disappearing.

\subsection{2. $M I P S 70 \mu m$ Colors}

Figure [6] shows the distribution of [24]-[70] colors for stars detected at $70 \mu \mathrm{m}$. All of these stars have spectral types later than K0 and have substantial emission at both $24 \mu \mathrm{m}$ and $70 \mu \mathrm{m}$. The right panel of Figure 6 shows that the stars with $70 \mu \mathrm{m}$ detections include 
both 'anemic' and 'thick' sources, indicating that at least some stars with dust-poor inner regions may have very luminous outer disks. While the distribution of MIPS colors appear correlated (stronger $24 \mu \mathrm{m}$ emission $\rightarrow$ stronger $70 \mu \mathrm{m}$ emission), the small number of stars with $70 \mu m$ detections precludes any robust statistical analysis to determine if this trend is real.

To provide a context for our $70 \mu m$ detected stars, we compare their colors to those for two stars in the $\eta$ Cha Association using data from Gautier et al. (2008). Even though $\eta$ Cha is substantially older than IC 348 ( $~ 8 \mathrm{Myr})$, its proximity to the Sun and lack of high IR background allow for many $70 \mu m$ detections; the median Taurus SED currently does not include a $70 \mu \mathrm{m}$ data point. Overplotted as diamonds are colors for ECH-15 (top center), an accreting late-type star with a primordial disk, and ECH-2 (lower left), which has weak, optically-thin emission consistent with a debris disk. The MIPS colors of IC 348 stars are slightly redder than ECH-15 at both $24 \mu \mathrm{m}$ and $70 \mu \mathrm{m}$ and far redder than ECH-2.

\subsection{SEDs of new MIPS Detections}

\subsubsection{Constraints on Disk Properties of Sources with MIPS-70 $\mu m$ Detections}

The addition of the MIPS-70 $\mu m$ data better determines plausible circumstellar disk properties from models with nearly identical fluxes through $24 \mu \mathrm{m}$ but very different farIR fluxes. To better constrain the disk morphology of IC 348 sources, we compare the SEDs of MIPS-70 $\mu \mathrm{m}$-detected sources with the grid of 3D radiative transfer models from Robitaille et al. (2006, 2007). In fitting the SEDs, we assume a $10 \%$ uncertainty in the IRAC and MIPS-24 $\mu \mathrm{m}$ fluxes and a 20\% uncertainty in the MIPS-70 $\mu \mathrm{m}$ fluxes to account for photometric error, zero-point uncertainty, and variability. We allow for a range of distances $(\sim 300-350 \mathrm{pc})$ and a range of optical extinction, typically comparable to the standard deviation in the extinction as determined by Muench et al. (2007). The online fitting tool then returns the SEDs of the best-matching models and lists the disk characteristics (inner and outer disk radius, accretion rate, disk mass, disk flaring index, etc.) of the models.

Figure 7 shows the fitted spectral energy distributions. In each panel, we overplot the SED of the best-fit bare stellar photosphere (dotted line), the best-fit SED model (solid black line), and other SED models with $\chi^{2}-\chi_{\text {best }}^{2}<10$. The $\chi_{\text {best }}^{2}$ for the sources range from $\chi^{2}=9.4$ (ID 103542) to $\chi^{2}=136$ for ID 67 . While the $\chi^{2}$ value for the latter source is large, much of the power in the $\chi^{2}$ values comes from measurements at shorter wavelengths (e.g. J band) where the photometric uncertainty is very small.

One source, ID-51, has a nearly flat $\operatorname{SED}(\lambda \mathrm{F} \lambda)$ from $2 \mu m$ through $70 \mu m$ and is thus 
consistent with "flat-spectrum" protostars like those found in Taurus-Auriga (Kenyon and Hartmann 1995). Such sources may be in the process of dispersing their protostellar envelopes (Calvet et al. 1994). Parameters for the best-fit model include a flaring index of $\beta=1.1(\beta=\mathrm{H} / \mathrm{r}$, where $\mathrm{H}$ is the disk scale height) and a disk mass of $\sim 3 \times 10^{-2} \mathrm{M}_{\odot}$. The SED of ID-51 rises from I band through $5.8 \mu \mathrm{m}$, which is consistent with a $360 \mathrm{~K}$ protostar as suggested by Muench et al. (2007).

Half of the $70 \mu \mathrm{m}$ sample - IDs 13, 10343, and 10352 - have SEDs consistent with premain sequence stars surrounded by primordial disks. These sources have clear IR excesses at near-IR wavelengths and SEDs whose flux declines with wavelength from peak values at $\sim 1-2 \mu \mathrm{m}$. Assuming a standard grain size distribution $\left(\mathrm{n}(\mathrm{a}) \propto \mathrm{a}^{-3.5}\right)$ and a solar gasto-dust ratio, these sources have disks with masses $\sim 10^{-3}-10^{-2} \mathrm{M}_{\odot}$, comparable to the Minimum Mass Solar Nebula. The disk flaring indices range from $\beta=1.01$ (for ID 13) to 1.17 (ID 10343). While Muench et al. (2007) identify ID-13 as a protostar, its IRAC slope is slightly steeper than their criteria for either protostars or flat spectrum sources. Our analysis coupled with its published spectral type $\left(\mathrm{M} 0.5 ; \mathrm{T}_{e} \sim 3800 \mathrm{~K}\right)$ indicates that it may be at the boundary between a flat spectrum source and a pre-main sequence star + primordial disk.

Two sources, IDs 31 and 67, show evidence for a strong flux deficit from $3.6 \mu \mathrm{m}$ through 8 $\mu \mathrm{m}$ but substantial emission at both $24 \mu \mathrm{m}$ and $70 \mu \mathrm{m}$. Disk parameters from the best-fitting models have a wide range of disk masses $\left(10^{-7}-10^{-2} \mathrm{M}_{\odot}\right)$ but typically have large inner disk boundaries ( $\sim 1-50 \mathrm{AU})$. While a depleted mass in warm dust and inner holes are consistent with disks in an intermediate stage between primordial and debris -'evolved' primordial disks or, as typically described, 'transitional' disks- there are alternate possibilities. Specifically, given that IC 348 contains many protostars, the strong 24 and $70 \mu \mathrm{m}$ emission could be due to a protostar lying in close proximity to IDs 31 and 67 . Because such sources would be unresolved with MIPS and too faint to detect from optical/near-to-mid IR imaging, their presence cannot be ruled out. However, these two sources are located in regions well away from most IC 348 protostars identified by Muench et al. (2007).

\subsubsection{Evolutionary States of Disks with New MIPS-24 $\mu \mathrm{m}$ Detections}

In this section, we investigate the evolutionary states of disks surrounding sources with new MIPS-24 $\mu \mathrm{m}$ detections. To provide a simple but effective probe of the disks' evolutionary states, we compare their SEDs to the median Taurus SED and upper/lower quartiles of Taurus sources and debris disk SEDs. We use the Taurus SED and upper/lower quartiles from Furlan et al. (2006); for the debris disk SED, we adopt the synthetic SED of a warm, terrestrial planet-forming debris disk around a solar-mass star from Kenyon and Bromley 
(2004). Evolutionary states derived from these simple comparisons show good agreement with those obtained from more detailed SED modeling (see Currie et al. 2009).

Following Currie et al. (2009), we classify sources as having primordial disks if their 3.6 $\mu m$ to $24 \mu m$ emission is comparable to or greater than the lower quartile of the median Taurus SED. Sources lacking clear excess emission in the IRAC bands but emission comparable to Taurus at $24 \mu \mathrm{m}$ are identified as disks with inner holes. Sources with IRAC and MIPS excess emission significantly weaker than the lower quartile Taurus SED but stronger than the debris disk SED are labeled 'homologously depleted disks' (Currie et al. 2009). Here, we define "significantly weaker" as less than half of the lower-quartile Taurus SED flux at 24 microns. This stricter criterion is justified because, unlike in Currie et al. (2009), most (68\%) of the new detections are stars later than M3, which are intrinsically redder and fainter than the K7-M2 stars used to derive the median Taurus SED, and thus should have systematically weaker primordial disk emission. Finally, sources with IR excess emission comparable to the debris disk SED are identified as debris disk candidates.

Figure 8 shows SEDs of sources representative of each disk class. All source SEDs are labeled by their ID number, J2000 coordinates, and extinction. We also include the IRAC slope (stron/weak IRAC, photosphere) following the formalism of Lada et al. (2006) as modified by Currie et al. (2009). The figures are arranged to illustrate the evolution of primordial disks (top panel) into debris disks (bottom panel) along two separate pathways: homologously depleted disks (second and third row panels, lefthand side) and disks with inner holes (second and third row panels, righthand side) (see Lada et al. 2006; Currie et al. 2009). The Appendix contains the atlas of all SEDs; Table 5 lists results for all stars. Below we describe the frequency and characteristics of each disk class. Because of a lack of short wavelength data, we did not determine the evolutionary states for IDs 746 and 2096.

1. Primordial Disks- Nine sources have SEDs indicative of primordial disks. The levels of disk excess from most of these sources are more comparable to the lower quartile of the median Taurus SED than the median Taurus SED. However, most sources in IC 348 have spectral types later than M4; it is not clear whether their slightly weaker emission is an evolutionary effect or simply a temperature (contrast) effect (Ercolano et al. 2009) because the median Taurus SED was derived from hotter (K7-M2) stars. Some of these sources have mid-IR emission comparable to the median Taurus SED, a characteristic consistent with a relatively unevolved primordial disk.

2. Homologously Depleted Disks- Seven sources have disks that produce a clear IR excess from $\sim 4.5 \mu \mathrm{m}$ to $24 \mu \mathrm{m}$ but at a level significantly weaker than Taurus: a 'homologously depleted' disk (Currie et al. 2009). Because both the IRAC and MIPS 
emission is depleted by up to a factor of 10 relative to the Taurus SEDs, it is unlikely that this weaker emission is simply due to flatter disk geometries. The weaker emission is likely due in part to significantly lower warm dust masses which can emerge from a combination of grain growth and accretion onto the star (see Currie et al. 2009). The SEDs exhibit a power-law decline with $\lambda \mathrm{F}_{\lambda} \propto \lambda^{-q}$, where $\mathrm{q} \sim 1.5-2.5$.

3. Disks with Inner Holes - Two sources show evidence for a disk with little or no emission in the IRAC bands but substantial MIPS emission, up to a level comparable to the median Taurus SED. These properties are characteristic of disks with inner regions mostly cleared of material. Because these disks represent only one of the two pathways from primordial disks to debris disks, and thus comprise one of two types of transitional disks, (Currie et al. 2009; Lada et al. 2006), we do not equate them with transitional disks but call them 'disks with inner holes' instead.

4. Debris Disks Candidates - Five sources have MIPS emission comparable to predicted fluxes from debris disks. This emission is a factor of $\approx 100$ weaker than primordial disks and is also weaker than emission from homologously depleted disks, and disk with inner holes. We label these sources 'debris disk candidates'.

Debris disks are usually defined as disks with second-generation dust, a property that correlates with broadband SEDs but cannot be directly inferred from SEDs. Disks around late type, low-mass stars have a much lower contrast with the photosphere than do disks around high-mass stars; thus, there is less difference in broadband fluxes between debris disks and disks in earlier evolutionary stages. Some of these disks could be very evolved (homologously depleted, inner hole) disks with first generation dust, not second generation dust. Others have larger photometric errors at $24 \mu \mathrm{m}$ and could be bare photospheres instead. Thus, our labeling of these sources as 'debris disk candidates' is tentative and requires more analysis for confirmation. We consider these sources further in $\S 3.4$.

5. Stars Without Disks - Two sources have SEDs that are consistent with bare stellar photospheres. ID-47, a K4 star, is the source that most obviously lacks IR excess emission. It lacks any IRAC-excess emission, and its $24 \mu \mathrm{m}$ flux is slightly bluer than a stellar photosphere. However, this source has a marginal, $5 \sigma$ detection, making its flux consistent with a stellar photosphere to within $\sim 2 \sigma$. ID-49 also has a very marginal IR excess at $24 \mu \mathrm{m}\left(\mathrm{K}_{s^{-}}[24] \sim 0.5\right)$ that is within $2 \sigma$ of photospheric colors. Thus, there is no clear evidence for a disk surrounding this star. 


\subsubsection{Mid-IR Colors of Sources with Disks in Various Evolutionary States}

In Figure 9 (top panel), we show the dereddened $\mathrm{K}_{s^{-}}[8]$ vs. $\mathrm{K}_{s^{-}}[24]$ color-color diagram for sources with new MIPS-24 $\mu m$ detection 1 . Symbols for sources with new MIPS detections correspond to their derived disk evolutionary state: primordial disks (circles), disks with inner holes (triangles), homologously depleted disks (inverted triangles), and debris disk candidates/photospheres (squares). Sources with primordial disks lie redward of a region defined by a vertical and horizontal short-dashed line. Polygonal regions enclose the range of colors for the two types of transitional disks: homologously depleted disks (dash-three dots) and disks with inner holes (dashes). Debris disk candidates and photospheres comprise all the newly-detected sources bluer in $\mathrm{K}_{s^{-}}[24]$ than the homologously depleted/inner hole boundaries. The positions of sources later than K0 in IRAC/MIPS color-color diagrams thus show a good separation by disk evolutionary state as determined in the previous section.

For reference, we overplot the colors of median Taurus SED with upper and lower quartiles (asterisks). The median Taurus SED $\left(\mathrm{K}_{s^{-}}[8]=2.1, \mathrm{~K}_{s^{-}}[24]=5.1\right)$, the upper quartile $\left(\mathrm{K}_{s^{-}}[8]=2.5, \mathrm{~K}_{s^{-}}[24]=6.3\right)$, and lower quartile $\left(\mathrm{K}_{s^{-}}[8]=1.8, \mathrm{~K}_{s^{-}}[24]=4.8\right)$ all lie within the primordial disk region. All of the sources identified as having primordial disks have 8 (24) $\mu m$ fluxes (measured relative to K-band) a factor of $\gtrsim 1.6(2.1)$ smaller than the lower quartile Taurus SED or $24 \mu \mathrm{m}$ fluxes reduced by a factor of $\approx 2.1$. The population from which the median Taurus SED was constructed includes sources with inner holes (e.g. DM Tau) or a significantly low dust mass (V836 Tau), so a median SED constructed only from sources with full disks has redder colors. Therefore, the fact that the boundaries enclosing primordial and non-primordial disks in Figure 9 (top panel) are bluer than positions of the Taurus SED colors indicates that our identification of non-primordial disks is conservative.

We also overplot IC 348 sources with previous MIPS detections from Lada et al. (2006) as filled dots with a brightness corresponding to their IRAC slope: 'thick' sources (light-grey dots), 'anemic' sources (grey dots), and 'diskless' sources (black dots). There is generally a good correspondance between the IRAC slope and the inferred evolutionary states as defined by the colors (e.g. diskless=photospheres/debris, anemic=homologously depleted/inner holes, thick=primordial). However, some 'thick' sources are located in the region corresponding to homologously depleted disks and some 'anemic' sources are located in the region corresponding to primordial disks.

Figure 9 (bottom panel) shows the dereddened $\mathrm{K}_{s^{-}}[8]$ vs. $\mathrm{K}_{s^{-}}[24]$ diagram for sources earlier than K0, which for a 2-3 Myr-old cluster corresponds to stars with masses $\gtrsim 1-2$

\footnotetext{
${ }^{1}$ Two sources - IDs 746 and 2096 - lack 2MASS K $\mathrm{K}_{s}$ detections and are thus not shown.
} 
$\mathrm{M}_{\odot}$ (Baraffe et al. 1998; D’Antona and Mazzitelli 1994). There is no fiducial SED (and thus no empirical colors) characterizing primordial disks surrounding stars of this mass range. To provide a context for the colors of these IC 348 sources, we overplot the colors of a F9 star with a massive debris disk, $\mathrm{h}$ and $\chi$ Per-S5 (small asterisk, Currie et al. 2007b), and AB Aurigae (large asterisk), an A0 star in Taurus surrounded by a primordial disk. The colors for AB Aur are estimated from the 2MASS and Spitzer-IRS fluxes at $2.2 \mu m, 8$ $\mu m$, and $24 \mu m$ (Furlan et al. 2006). ID-4 has colors much bluer than $\mathrm{h}$ and $\chi$ Per-S5 and is thus consistent with being a debris disk candidate. Among previously-detected sources, one (ID-8012) clearly has primordial disk emission $\left(\mathrm{K}_{s^{-}}[8] \sim 3.4, \mathrm{~K}_{s^{-}}[24] \sim 5.3\right)$. Five others with $\mathrm{K}_{s^{-}}[24]=2.5-5.5$ require further analysis because their mid-IR colors could be consistent with either a debris disk or a remnant protoplanetary disk (i.e. homologously depleted, inner hole).

In summary, the dereddened $\mathrm{K}_{s^{-}}[8]$ vs. $\mathrm{K}_{s^{-}}[24]$ color-color diagram separates late-type IC 348 sources by disk evolutionary state. This analysis is more ambiguous for early/intermediate spectral type stars. Sources of any spectral type identified as debris disk candidates on the basis of their mid-IR colors require further analysis because debris disks, by definition, lack gas accretion and have second generation dust. These properties cannot be determined from mid-IR colors alone. In the next section, we will investigate the properties of sources with ambiguous states, including all debris disk candidates. After determining their likely evolutionary states, we will use the results from color-color diagrams presented in this section to analyze the global properties of the IC 348 disk population.

\subsection{Evolutionary State of Disks Surrounding Early/Intermediate-Type Stars and All Debris Disk Candidates}

Many early/intermediate spectral type sources with mid-IR colors bluer than those harboring primordial disks (e.g. AB Aurigae) have ambiguous disk evolutionary states. Many sources surrounding late type, low-mass stars with new MIPS-24 detections have extremely weak mid-IR disk emission that could either come from a debris disk or a remnant protoplanetary disk. In this section, we constrain the evolutionary state of disks surrounding stars from these two populations. We first use new and archival optical spectra to identify accretors and to remove them from the population of debris disk candidates. For remaining debris disk candidates, we estimate the mass of emitting dust (through $24 \mu \mathrm{m}$ ) and calculate dust removal timescales. The early/intermediate type stars investigated in this section are IDs 2-8, 11, 19-20, 25, and 30-31. The late-type stars investigated here, all of which have new MIPS-24 $\mu m$ detections, are IDs 39, 53, 56, and 1939. 


\subsubsection{Accretors}

Using new and archival optical spectra, we can remove several accreting early-type stars from the list of debris disk candidates. These include ID 19 - identified as an accretor from this work - and IDs 5 and 31, which were previously identified as accretors. None of the late-type stars show unambiguous evidence for accretion based on their $\mathrm{H}_{\alpha}$ equivalent widths.

One source -ID 2 - is a borderline case. Our spectra for ID-2 may exhibit slightly weakened $\mathrm{H}_{\alpha}$ absorption line compared to non-accreting stars of the same spectral type (ID8). Based on unpublished high-resolution echelle spectroscopy, Dahm (2008) identifies this star as having an $\mathrm{H}_{\alpha}$ emission line reversal plausibly due to accretion. Thus, we remove it from our sample of debris disk candidates. We treat this star as lacking evidence for circumstellar gas, though we caution that accretion may persist at low rates. Given that the presence or absence of accretion is a primary marker of disk evolutionary state, the inability to rule out or identify accretion for this source renders its disk status uncertain.

\subsubsection{SED Modeling of Debris Disk Candidates: Estimating Dust Masses and Temperatures}

Next, we compare the SEDs of early-to-late type debris disk candidates with 3D radiative transfer disk models for a range of disk masses. To model the near-to-mid IR emission of debris disk candidates, we compare their SEDs to the grid of 200,000 radiative transfer models from Robitaille et al. (2006), which include a range of spectral types and dust masses. The infrared fluxes predicted for disks are sensitive to the dust masses down to $\sim 10^{-10} \mathrm{M}_{\odot}$. By selecting the best-fitting SEDs, we estimate the minimum mass of small $(\lesssim 100 \mu m-1 \mathrm{~mm})$ dust grains required to reproduce the observed disk emission. We compare this estimate to typical dust masses in debris disks and primordial disks. Typical dust masses for debris disks range from $10^{-11} \mathrm{M}_{\odot}$ to $5 \times 10^{-9} \mathrm{M}_{\odot}$ (cf. Chen et al. 2005; Low et al. 2005). Dust masses 2 typically needed to reproduce the IRAC and MIPS excess emission for transitional disks range from $10^{-7}-10^{-5} \mathrm{M}_{\odot}$ (Currie et al. 2009).

We choose a representative sample of sources to model: IDs 6, 8, 20, and 30 for the early/intermediate-type population and IDs 39 and 1939 for the late-type population. Because most of the other sources have weaker disk emission or comparable emission, the

\footnotetext{
${ }^{2}$ This mass is not the same as the disk masses, which is more typically cited. To derive the disk mass from the dust mass, Andrews and Williams (2005) and other other authors assume a gas to dust ratio of 100. The Robitaille et al. (2006) models assume the same gas to dust ratio.
} 
required mass of emitting dust in their disks is most likely comparable or lower. Therefore, modeling the SEDs of these sources to estimate the mass of small, emitting dust grains is sufficient to yield approximate dust masses for all debris disk candidates.

Figure [10 shows SED model fits to the six sources. Table 6] summarizes the model parameters for each source. The best-fit Robitaille et al. (2006) models accurately reproduce the observed fluxes from these sources. Assuming a gas-to-dust ratio of 100, the required disk masses for the best-fit Robitaille et al. models are $\sim 2-8 \times 10^{-8} \mathrm{M}_{\odot}$. The extremely low disk masses are consistent with the disks being very optically thin. The required masses in dust are $\approx 10^{-10}-10^{-9} \mathrm{M}_{\odot}$. These dust masses are lower than typical dust masses of primordial disks but similar to debris disk masses. Unless these sources had enormous reservoirs of extremely cold dust, which would not significantly contribute to the observed IRAC and MIPS $24 \mu \mathrm{m}$ emission, the total mass of dust in small grains is also likely much less than typical dust masses for primordial disks.

We can place further constraints on the temperature and luminosity of the dust. Most debris disk candidates have clear excess emission only at $24 \mu \mathrm{m}$ with dust temperatures colder than $200 \mathrm{~K}$ (Carpenter et al. 2009). However, IDs 6 and 8 have $8 \mu \mathrm{m}$ excess emission, which indicates that their dust, if confined to a narrow ring like in a debris disk, is warmer. Indeed, a single temperature blackbody fit to the SEDs of these sources yields dust temperatures of $400 \mathrm{~K}$ and $270 \mathrm{~K}$. These temperatures are similar to those for known terrestrial planetforming debris disks such as h and $\chi$ Per-S5, EF Cha, and HD 113766A (Currie et al. 2007b; Rhee et al. 2007a; Lisse et al. 2008). The fractional infrared luminosities $\left(\mathrm{L}_{D} / \mathrm{L}_{\star}\right)$ of debris disk candidates from one and two-temperature blackbody fits range from $5 \times 10^{-3}$ (ID-6) to $10^{-4}$ (ID-3). These fractional luminosities are two to three orders of magnitudes less than those for primordial disk-bearing stars but very similar to the stars with warm, terrestrial planet-forming disks as well as those with colder debris disks (e.g., $\beta$ Pic Rebull et al. 2008).

\subsubsection{Dust Dynamics and Removal Timescales}

To provide better constraints on the evolutionary state of debris disk candidates, we investigate dust dynamics. If the dust must be second generation, then the disks are debris disks (Backman and Paresce 1993). We determine grain removal timescales in optically thin conditions in two cases: where there is a significant amount of undetected circumstellar gas (1-10 $\left.\mathrm{M}_{\oplus}\right)$ and where there is little or no gas. If the timescale for removal is much less than the age of the star in either case, the grains require a replenishment source through collisions between larger bodies. Disks around such stars would then fulfill the standard definition of a debris disk (e.g. Backman and Paresce 1993). 


\section{- Case 1: Optically-Thin Disk with Residual Circumstellar Gas}

Takeuchi and Artymowicz (2001) extensively investigated the dynamics of circumstellar dust in optically-thin disks with residual gas. Under these conditions, the dust grains migrate outward at a rate primarily determined by $\beta$, the ratio of the force from radiation pressure to the gravitational force:

$$
\beta=\frac{3 L_{\star}<Q_{p r}(a)>}{16 \pi G M_{\star} c s \rho_{s}} .
$$

When $\beta \geq 0.5$ the dust is removed from the system entirely on orbital timescales, which are $\approx 100-1000$ yr (see also Strubbe and Chiang 2006). Removal occurs independent of whether there is residual gas left in the disk (Takeuchi and Artymowicz 2001, Takeuchi 2008, pvt. comm.). Equation 1 can be rearranged to find the size of dust grain below which radiation pressure rapidly removes dust from the system (see also Backman and Paresce 1993; Burns, Lamy and Soter 1979):

$$
s_{\text {max }, \mu m}<1.14 \mu m\left(\frac{L_{\star}}{L_{\odot}}\right)\left(\frac{M_{\odot}}{M_{\star}}\right)\left(\frac{1 \mathrm{gcm}^{-3}}{\rho_{s}}\right)<Q_{p r}>.
$$

Typically, the sizes of mid-IR emitting dust grains range from $\approx 1 \mu \mathrm{m}$ to $10 \mu \mathrm{m}$ (e.g. Augereau et al. 1999; Furlan et al. 2006).

For early-type stars listed in Table 6, $\mathrm{s}_{\max }$ ranges from 2.2 to $17.8 \mu \mathrm{m}$, assuming $<Q_{p r}>\approx 1$ and $\rho_{s} \approx 1 \mathrm{~g} \mathrm{~cm}^{-3}$. Using a more realistic dust grain density of $\sim$ $0.1 \mathrm{~g} \mathrm{~cm}^{-3}$ (e.g. Augereau et al. 1999) essentially removes all dust grain sizes that can plausibly produce IRAC and MIPS disk emission. Grains responsible for producing this emission are blown out; mid-IR emission ceases unless the new grains are produced from collisions between larger bodies.

Dust grains much larger than $\mathrm{s}_{\max }(\beta<0.5)$ can produce 8-24 $\mu \mathrm{m}$ broadband excess emission in these early type stars and must also be second generation. For a 1-10 $\mathrm{M}_{\oplus}$ gas disk around an early-type star, the combined influence of radiation pressure, Poynting-Robertson drag, and gas drag push $\approx 10-100 \mu m$-sized dust from 10 AU to $\sim 300 \mathrm{AU}$ in $\approx 600-60,000 \mathrm{yr}$ (Takeuchi and Artymowicz 2001). For the early-type stars in Table 6, 120-200 K dust is located at $\approx 5-35$ AU. If pushed to $\sim 300 \mathrm{AU}$, the dust temperature drops by up to a factor of 5 , making the dust too cold to emit strongly at $24 \mu \mathrm{m}$. If new dust at 5-35 AU is not produced, then the disks' 8-24 $\mu \mathrm{m}$ emission will become undetectable. Thus, the observed mid-IR emitting dust must be second generation.

The effect of radiation pressure on dust surrounding late-type stars is less clear. The maximum grain size for ID-39 is $\sim 1.5 \mu \mathrm{m}$, a value that is slightly smaller than $\mathrm{s}_{\max }$ for 
the earlier-type stars. However, s $_{\max }$ for ID-1939 (and, by inference, all M stars that are debris disk candidates) is $\sim 0.98 \mu \mathrm{m}$, which begins to approach the sizes of large grains in the interstellar medium. While radiation pressure may be able to remove grains with these sizes, it is probably ineffective at completely removing grains with sizes typical of circumstellar disks (up to $10 \mu \mathrm{m}$ ) unless the grains are extremely porous. Therefore, the dust responsible for $24 \mu m$ disk emission is not necessarily removed from the disk by radiation pressure. However, grains with sizes typical of circumstellar disks and and densities of $\rho_{s} \approx 1 \mathrm{~g} \mathrm{~cm}^{-3}$ have $\beta$ up to 0.3 and could be transported from their original locations to $\approx 300$ AU scales (Takeuchi and Artymowicz 2001).

\section{- Case 2: No Gas or Trace Amounts of Gas}

In the absence of residual circumstellar gas, the dust dynamics are more simple. If $\beta$ for a given dust size is greater than 0.5 , then dust is rapidly removed by radiation pressure as in Case 1. If $\beta$ is $<0.5$ then Poynting-Robertson drag is the dominant removal mechanism. For $\rho_{s} \sim 1 \mathrm{~g} \mathrm{~cm}^{-3}$ and $\left\langle Q_{a b s}>\sim 1\right.$, the P-R drag timescale can be parameterized (e.g. Backman and Paresce 1993; Augereau et al. 1999):

$$
t_{P-R} \sim 7 \times 10^{4}\left(\frac{L_{\odot}}{L_{\star}}\right)\left(\frac{s}{1 \mu m}\right)\left(\frac{r}{10 A U}\right)^{2} y r
$$

where $\mathrm{s}$ is the grain size.

To derive the drag timescale for grains, we consider typical parameters for $\mathrm{r}_{A U}$ (the grain's distance from the star), $\left\langle Q_{a b s}>\right.$ (the absorption coefficient), and $\rho_{s}$ (grain volume density). Most sources have weak or negligible $8 \mu \mathrm{m}$ excesses and stronger 24 $\mu m$ excesses; thus, their dust temperatures are probably $\lesssim 125-250 \mathrm{~K}$ (Currie et al. 2008a). While $120 \mathrm{~K}$ dust can produce a strong $24 \mu \mathrm{m}$ excess but weak/no $8 \mu \mathrm{m}$ excess, colder dust with $24 \mu \mathrm{m}$ emission further down the Wien tail can also produce these trends. Given the lack of constraints on dust's temperature and location, we calculate $\mathrm{t}_{P-R}$ for a range of distances $-1,10$, and $30 \mathrm{AU}$ - which cover the range of distances for dust at 100-200 K for most stars listed in Table 6 assuming simple radiative equilibrium.

As shown in Table 6, P-R drag removes small grains surrounding early-type IC 348 on much timescales shorter than the $\sim 2-3$ Myr age of the cluster. For $1 \mu m$ grains, the removal timescales range from being several hundred years to $\sim 100,000$ years for IDs $6,8,20$, and 30 . In all cases, the removal timescale is less than $\approx 1 / 20$ th of the median age for IC 348 stars. Thus, without a replenishment source, dust grains surrounding these stars are removed rapidly, on timescales much less than the age of the star.

The effect of P-R drag on dust surrounding late-type stars is less clear. If the dust orbits closely to the stars (1 AU), then P-R drag can remove the dust on rapid timescales. 
However, for both ID-39 and ID-1939, it is not clear that the dust requires a replenishment source if it is located at much larger distances (30 AU). For ID-1939, dust at intermediate distances (10 AU) could also be remnant protoplanetary dust which are plausible. The Robitaille et al. (2006) models that best fit the IDs 39 and 1939 SED assumes inner hole sizes of $\approx 100 \mathrm{AU}$ and $31 \mathrm{AU}$, respectively. Thus, the dust need not be second generation.

In summary, the dust grains surrounding the AFG stars listed in Table 6 must be 2nd generation. This result is independent of whether the disk has no gas or has residual gas. Because the dust must be 2nd generation, disks surrounding these stars must be debris disks. Other characteristics of these disks - lack of gas accretion and minimum required dust mass support this conclusion. The only situation in which the dust can be primordial is if the disk retains an enormous reservoir of gas, $>>100-1000 \mathrm{M}_{\oplus}$. In such a case, the gas-dust coupling is strong enough that the removal timescale from radiation pressure may be comparable 1-3 Myr (Takeuchi, pvt. comm.). However, the extreme gas to dust ratios implied by these conditions, $>>10^{8}-10^{9}$, would be highly unusual if not unphysical. Spectroscopy of sources listed in Table 6 fail to identify any with active gas accretion, which would be expected if the disks had an enormous reservoir of gas left. By far the most simple explanation is that these sources are very gas poor or gas free. The dust responsible for their MIPS excesses then most likely requires a replenishment source: collisions between larger bodies. Thus, these disks fit the standard definition of a debris disk.

In contrast, it is not clear if dust surrounding late type stars (e.g. ID-39 and ID-1939) must be second generation. Both ID-39 and ID-1939 lack evidence for accretion and have a low disk mass. However, for ID-39 it is not clear that its dust must second generation if the dust is located far from the star and is large $(\beta<0.5)$. The dust surrounding ID-1939 need not be second generation if it is located beyond $\approx 1 \mathrm{AU}$ of the star and or is entrained in a substantial reservoir of gas $\left(\gtrsim 1 \mathrm{M}_{\oplus}\right)$. Both of these situations are at least plausible in principle. Other removal mechanisms such as corpuscular wind drag (Plavchan et al. 2005, 2009) may be important for late-type, low-mass stars are neglected here. Analyzing dust removal timescales after including these effects, may affect our conclusion that the dust surrounding late-type stars need not be from a debris disk.

Based on the above analysis, we classify all AFG stars with characteristics typical of those listed in Table 6 (mid IR colors, lack of accretion signatures) as debris disk candidates. Debris disk candidates surrounding $\mathrm{K}$ and $\mathrm{M}$ stars with similar characteristics could be either debris disks or evolved primordial disks/transitional disks with very low dust masses. To be 
conservative, we classify them as homologously depleted disks 3 .

\section{Global Analysis of the IC 348 Disk Population}

We now combine our new optical spectroscopy and MIPS photometry of IC 348 stars with archival data to investigate the properties of the entire IC 348 disk population. Our analysis has several goals. To investigate the frequency of gas accretion as a function of spectral type, we combine our analysis of optical spectra with archival spectroscopic data. We consider whether the frequency of signatures of circumstellar gas depends on the spectral type of stars, a trend consistent with a stellar-mass dependent dispersal of gas disks.

Second, we investigate how the rate of dust evolution varies with stellar mass. For a simple, empirical probe of disk evolution, we first analyze the $\mathrm{K}_{s^{-}}$[8] and $\mathrm{K}_{s^{-}}[24]$ colors as a function of spectral type and IRAC slope. We consider the evidence for a spectral-type dependent dust luminosity at $8 \mu \mathrm{m}$ and $24 \mu \mathrm{m}$. To further see whether disks surrounding stars of different stellar masses evolve at different rates, we conclude by constraining the relative frequency of disks in different evolutionary states 4 .

\subsection{Gas Disk Evolution as a Function of Stellar Mass}

To investigate gas disk evolution in IC 348, we analyze data presented in this work, archival data summarized in Lada et al. (2006), and data from Dahm (2008). We use empirical criteria to identify accretors (primarily the $\mathrm{H}_{\alpha}$ equivalent width) from White and Basri (2003) for stars later than G0. Stars with very early spectral types (BAF) have a deep $\mathrm{H}_{\alpha}$ absorption lines produced from the stellar photosphere. For these stars, we use our results and those from Dahm (2008) to identify stars with $\mathrm{H}_{\alpha}$ emission line reversals consistent with accretion. Data for late-type stars summarized in Lada et al. (2006) typically draw from Luhman et al. (2003) and Luhman (1999). Some sources lacking $\mathrm{H}_{\alpha}$ equivalent widths from optical spectroscopy have near-IR spectra that either confirm their status as accretors (Luhman et al. 2005; Dahm 2008) or rule out clear evidence of accretion (Dahm 2008). We include these sources in our statistics. We remove from the sample all other stars lacking

\footnotetext{
${ }^{3}$ While the disk models best fitting the SEDs have large inner holes, the low disk vs. photosphere contrast afforded by $\mathrm{K}$ and $\mathrm{M}$ stars means that the disk mass may simply be extremely low (Ercolano et al. 2009).

${ }^{4}$ We do not include sources that lack near-IR photometry because SED modeling of such sources (e.g., IDs 746, 2096) was unsuccessful in constraining their evolutionary states
} 
published $\mathrm{H}_{\alpha}$ equivalent widths.

Figure11(left panel) plots the $\mathrm{H}_{\alpha}$ equivalent width versus spectral type. Stars identified as accretors are shown as black dots while those lacking evidence for accretion are shown as grey dots. Stars without $\mathrm{H}_{\alpha}$ in emission that lack evidence for accretion are shown along a line corresponding to $\mathrm{EW}\left(\mathrm{H}_{\alpha}\right)=-15 \AA$ (in absorption). For reference, we draw lines corresponding to $\operatorname{EW}\left(\mathrm{H}_{\alpha}\right)=0$ (the division between emission and absorption) and $10 \AA$, which is the division typically used to distinguish between classical $\mathrm{T}$ Tauri stars and weak-line $\mathrm{T}$ Tauri stars. According to our criteria, several stars with spectral types earlier than K5 have $\mathrm{EW}\left(\mathrm{H}_{\alpha}\right) \leq 10 \AA$ but are identified as accretors, while others with spectral types later than M3 have $\mathrm{EW}\left(\mathrm{H}_{\alpha}\right) \geq 10 \AA$ but are not accreting.

Figure 11 (right panel) plots the frequency of accretors as a function of spectral type. We divide the sample into three spectral type bins: sources earlier than K3, between K3 and M0, and between M0 and M2.5. For a 2-3 Myr-old cluster, these divisions correspond to stars with masses $\gtrsim 1.4 \mathrm{M}_{\odot}, 0.8-1.4 \mathrm{M}_{\odot}$, and 0.5-0.8 $\mathrm{M}_{\odot}$ (Baraffe et al. 1998). Sources with probable masses $\gtrsim 1.4 \mathrm{M}_{\odot}$ have the lowest frequency of accretion $\left(5 / 29: 17.2^{+9}{ }_{-4.8} \%\right)$. Nearly half of the stars with probable masses of $0.8-1.4 \mathrm{M}_{\odot}$ and $0.5-0.8 \mathrm{M}_{\odot}$ show evidence for gas accretion $\left(7 / 17,41.2^{+12.2}-10.3 \%\right.$, and $18 / 41,43.9^{+7.8}-7.3 \%$, respectively).

In deriving these frequencies, we acknowledge that using $\mathrm{H}_{\alpha}$ as a diagnostic of accretion, even following the prescription of White and Basri (2003), is prone to some uncertainties. The contrast between the $\mathrm{H}_{\alpha}$ emission line and the photosphere is stronger for later type, lower-luminosity stars. The large number of stars later than $\sim$ M5 lacking optical spectra yields an uncertain frequency of accretors for the lowest-mass stars. Nevertheless, the observed trend of accretion frequency is consistent with the trend of infrared excess as a function of spectral type from Lada et al. (2006) at a $\sim 1-2 \sigma$ significance: the frequency is lowest for early type, high-mass stars and highest for $0.6-1 \mathrm{M}_{\odot}$ stars. The results are consistent with a stellar-mass dependent dispersal of gaseous circumstellar disks (see also Kennedy and Kenyon 2009).

\subsection{Circumstellar Dust Evolution as a Function of Stellar Mass}

To investigate the dust luminosity as a function of stellar mass, we compare the intrinsic $8 \mu \mathrm{m}$ and $24 \mu \mathrm{m}$ luminosity for each star relative to its intrinsic K-band luminosity. The dereddened $\mathrm{K}_{s^{-}}$[8] colors as a function of spectral type and IRAC slope are shown in the left panel of Figure 12, The figure includes photometric errors for each source along with the locus for photospheric colors from the Kurucz-Lejeuene stellar atmosphere models. The 
diskless, anemic, and thick sources are clearly separated into a blue to red sequence of colors across the entire range of spectral types. Diskless sources (black dots) have $\mathrm{K}_{s^{-}}$[8] colors ranging from $\sim 0$ to 0.2 from A0 to M6/M7. Anemic sources (grey dots) are slightly redder $\left(\mathrm{K}_{s^{-}}[8] \sim 0.2-1.25\right)$ and have weak excesses. Finally, thick sources (light grey dots) are systematically redder, with $\mathrm{K}_{s^{-}}[8] \sim 1-3$.

The dereddened $\mathrm{K}_{s^{-}}[8]$ color qualitatively shows the same separation into thick, anemic, and diskless classes as the observed $\alpha_{[3.6]-[8]}$ from Lada et al. (2006). Together with Lada et al. (2006), our results show that the median $8 \mu m$ excess is larger for later-type stars. This result suggests that inner disks around early type, high-mass stars evolve faster than around solar and subsolar-mass stars.

The distribution of dereddened $\mathrm{K}_{s^{-}}[24]$ colors (right panel) is more complicated. There is considerable overlap in $24 \mu \mathrm{m}$ excesses between sources with varying levels of $8 \mu \mathrm{m}$ excess, especially for stars with spectral types between M1 and M7. The 'thick' and 'anemic' stars in this spectral type range have a wide range (2-6 mag) of $24 \mu \mathrm{m}$ excesses.

The distribution of $\mathrm{K}_{s^{-}}[24]$ colors suggests that disks around later-type stars are more luminous relative to the star even though the contrast between the star and a disk of a given dust mass is likely lower for M stars (Ercolano et al. 2009). The fractions of MIPS-detected stars with strong $24 \mu \mathrm{m}$ excesses $\left(\mathrm{K}_{s^{-}}[24]>4\right)$ for stars earlier than $\mathrm{K} 3\left(>1.4 \mathrm{M}_{\odot}\right)$, between K3 and M0 (0.8-1.4 $\left.\mathrm{M}_{\odot}\right)$, and between M0 and M2.5 (0.5-0.8 $\left.\mathrm{M}_{\odot}\right)$ are 5/18 (27.8\%), 7/10 (70\%), and 20/24 (83.3\%).

Despite of the large number of stars lacking detections, this general trend clearly holds for stars with masses $\gtrsim 0.5 \mathrm{M}_{\odot}$. The $24 \mu \mathrm{m}$ upper limits for the 12 stars earlier than $\mathrm{K} 3$ without MIPS detections constrain the stars' excesses to be $\lesssim 3-4$ magnitudes. Assuming that all stars between K3 and M0 currently lacking MIPS detections (10) have weak excesses or no excesses, more than $35 \%$ of these stars must have strong excesses $(7 / 20)$, a percentage that is still much higher than that for earlier type, higher-mass stars $(4 / 31 ; 12.9 \%)$.

To compare the median level of excesses for high/intermediate to low-mass IC 348 stars, including sources with $24 \mu \mathrm{m}$ upper limits, we use the ASURV Survival Analysis package Rev 1.2 (Isobe and Feigelson 1986; LaValley et al. 1992). Because our goal is to compare the $\mathrm{K}_{s^{-}}[24]$ colors for two stellar mass bins, we treat the data as univariate and compare the two populations using the Gehan's General Wilcoxon Test (Permutated Variance and Hypergeometric Variance), the Peto-Prentice Generalized Wilcoxon Test, and the KaplanMeier Estimator. The three Wilcoxon tests yield the probability that the high/intermediate and low-mass populations are drawn from the same population. The Kaplan-Meier Estimator yields the likely median value of the $\mathrm{K}_{s^{-}}[24]$ colors for both populations while accounting 
for non detections. We divide the sample into stars with spectral types earlier than K5 and those with spectral types between K5 and M2.5. For a 2.5 Myr-old cluster, these spectral type ranges correspond to stars with $\mathrm{M}_{\star}>1 \mathrm{M}_{\odot}$ and $\mathrm{M}_{\star}=0.5-1 \mathrm{M}_{\odot}$ (Baraffe et al. 1998).

The ASURV statistical tests indicate that a) the excesses for high/intermedate mass and low-mass stars have statistically significant differences and b) the excesses are stronger for low-mass stars. The Wilcoxon tests find that the two populations have a probability of less than $0.14 \%$ (0.001-0.0014) of being drawn from the same parent population. The KaplanMeier estimator yields a small median $\mathrm{K}_{s^{-}}[24]$ color for high/intermediate-mass sources $\left(\mathrm{K}_{s}\right.$ $-[24] \sim 1.55 \pm 0.31$ ); the median color for low-mass sources is $\sim 3.19 \pm 0.40$. Very similar results are obtained if the division between samples is made anywhere from G0 to K5. Thus, the late type, low-mass IC 348 population has larger $24 \mu \mathrm{m}$ excesses from disks. Under the general picture that the luminosity of disks at a given wavelength decline with time, these results imply that disks around low-mass stars are less evolved than those around high/intermediate-mass stars.

For this analysis to be completely reliable, the censoring of the data must be 'random'. If nondetections are primarily due to high nebular background emission at the source positions then the censoring is random. If the sources lacking detections are intrinsically fainter, the censoring is not random About $56 \%$ and $51 \%$ of the stars with spectral types earlier than K5 and between K5 and M2.5 are detected at $24 \mu \mathrm{m}$. Because these frequencies are comparable, the censoring of data is very close to being random for stars earlier than M2.5. The frequency of $24 \mu \mathrm{m}$ detections drops substantially for stars later than M2.5 ( 30\%). Comparing the disk luminosities for $>0.5 \mathrm{M}_{\odot}$ stars with those for even lower-mass stars is then impeded by sample incompleteness for the latter population.

\subsection{Evolutionary State of Disks vs. Stellar Mass in IC 348}

We now consider the frequencies of disks in different evolutionary states as a function of stellar mass. For all stars, we quantify the evidence for disk emission at $24 \mu \mathrm{m}$. Figure 2 suggests that stars with $\mathrm{K}_{s^{-}}[24]$ colors $\gtrsim 1 \sigma\left(K_{s},[24]\right)$ away from the distribution of pho-

tospheric sources have significant excesses. Sources with smaller dereddened $K_{s^{-}}[24]$ colors are then identified as diskless photospheres. To guide our identification of primordial disks, transitional disks, and debris disks, we use the results from $\S 3.3 .3$ and $\S 3.4$. For stars later than K0, we use the semi-empirical division based on Figure 9 to identify primordial disks 
and transitional disks. For stars earlier than K0, we use the results of $\S 3.3 .3$ and $\S 3.4$ to select primordial disks, transitional disks, and debris disks.

Figure 13 shows the frequency of primordial disks, transitional disks, and debris disks as a function of spectral type/stellar mass. We divide the sample into the same three stellar mass bins used to compute the gas accretion frequency: $>1.4 \mathrm{M}_{\odot}$ (earlier than K3), 0.8-1.4 $\mathrm{M}_{\odot}(\mathrm{K} 3-\mathrm{M} 0)$, and $0.5-0.8 \mathrm{M}_{\odot}(\mathrm{M} 0-\mathrm{M} 2.5)$. We do not compute the relative disk fractions for later type, lower-mass stars where completeness at $24 \mu \mathrm{m}$ drops to $\approx 30 \%$.

Based on our analysis, the disk evolutionary states in IC 348 depend on stellar spectral type. Half of the MIPS-detected disks around intermediate/high-mass stars are likely debris disks (dashed line/diamonds). Primordial disks (solid line/crosses) comprise about 15\% of the total disk population. The MIPS-detected disk populations for stars with probable masses between $0.5 \mathrm{M}_{\odot}$ and $1.4 \mathrm{M}_{\odot}$ show very different distributions. Primordial disks comprise greater than $70 \%$ of the MIPS-detected disk population for stars in this mass range. The fact that stars in this mass range have (relative to the stellar photosphere) more luminous disks than their high/intermediate-mass counterparts is consistent with our determination that these stars typically have primordial disks.

The frequency of transitional disks (dotted line) for the MIPS-detected disk population ranges from $\sim 15 \%$ for $0.5-0.8 \mathrm{M}_{\odot}$ stars, to $20 \%$ for $0.8-1.4 \mathrm{M}_{\odot}$ stars, to $35 \%$ for high/intermediate-mass stars. This frequency is slightly higher than that computed for Taurus-Auriga $(<10 \%)$. Moreover, the transition disk frequency exceeds the frequency of primordial disks for high/intermediate-mass stars, a result similar to that found for solar/subsolar-mass stars in 5 Myr-old NGC 2362 (Currie et al. 2009). Depending on the nature of IC 348 sources currently lacking MIPS detections, mid-IR colors presented in Figure 12 indicate that the transition disk population for the lowest-mass stars $\left(\mathrm{M}_{\star}<0.5 \mathrm{M}_{\odot}\right)$ may also comprise a significant fraction of the total disk population $(\gtrsim 30 \%)$.

\section{Summary and Discussion}

\subsection{Summary of Results}

Using new optical spectra and new, deep MIPS $24 \mu \mathrm{m}$ and $70 \mu \mathrm{m}$ photometry we investigated the disk population of the 2.5 Myr-old IC 348 Nebula. Combining these data

\footnotetext{
${ }^{5}$ Within the transitional disk category, we include sources first identified in $\S 3.3 .3$ as debris disk candidates from Figure 9 because the dust around these sources need not be 2nd generation (see $§ 3.4 .3$ ). Separating these debris disk candidates from transitional disks does not qualitatively change our results.
} 
with previous work from Lada et al. (2006), we analyzed optical spectroscopic data for all stars, performed SED modeling, and examined the mid-IR colors of MIPS-detected members to constrain the disks' evolutionary states and probe how disk properties vary with stellar mass. Our study yields the following major results:

- IC 348 sources with MIPS-70 $\mu m$ detections include flat-spectrum protostars and pre-main sequence stars with optically-thick, luminous far IR disk emission. Some optically-thick disks show evidence for depleted inner disks; others do not.

- IC 348 stars with new and previous MIPS detections have disks in evolutionary states ranging from primordial disks to debris disks. In agreement with recent work (Lada et al. 2006; Hernandez et al. 2007; Currie et al. 2009), we find evidence for two separate pathways from primordial disks to debris disks. In homologously depleted disks, disks deplete their reservoir of small dust grains at all disk locations simultaneously. In a second sequence ('disks with inner holes'), disks clear their supply of small dust grains from the inside out.

- At $\mathrm{a} \sim 1-2 \sigma$ significance, signatures of circumstellar gas accretion are more frequent for solar and subsolar-mass stars than for high/intermediate-mass stars. This result is consistent with a gas disk dispersal timescale that is shortest for high/intermediatemass stars.

- The mid-IR disk luminosities of MIPS-detected disks are stellar-mass dependent. Relative to the stellar photosphere, $24 \mu \mathrm{m}$ emission from disks is lower for high/intermediatemass stars than for solar/subsolar-mass stars. If disks generally decline in luminosity as a function of time, this result implies that disks around high/intermediate-mass stars evolve faster.

- The evolutionary states of MIPS-detected disks are also stellar mass dependent. Most MIPS-detected disks surrounding high/intermediate-mass stars stars appear to be debris disks; primordial disks comprise only $\sim 15 \%$ of the disk population for $\mathrm{M}_{\star}>1.4$ $\mathrm{M}_{\odot}$. In contrast, most MIPS-detected disks around solar and subsolar-mass stars are primordial disks. For stars of all masses, transitional disks (homologously depleted or disks with inner holes) comprise $\sim 15-35 \%$ of the MIPS-detected disk population.

\subsection{The Evolutionary State of Anemic Disks}

Our SED modeling and investigation of the mid-IR colors of IC 348 stars clarifies the nature of anemic disks identified by Lada et al. (2006). Among late type stars, most anemic 
disks are homologously depleted disks or are disks with inner holes. Several anemic disks are probably primordial disks. Among earlier-type stars with probable masses $\gtrsim 1.4 \mathrm{M}_{\odot}$, anemic disks comprise a broader range of evolutionary states. At least two anemic disks (surrounding IDs 6 and 8) are probably debris disks. In contrast, ID-31 has strong mid-IR emission and gas accretion signatures more consistent with a transitional disk, specifically one with an inner hole.

Thus, as pointed out by Currie et al. (2009), a single IRAC slope serves as a useful first-order probe of disk properties, but a full analysis of IRAC and MIPS data combined with gas accretion signatures is required for an accurate taxonomy of disks. Our results indicate that a full analysis is especially necessary if the stellar population includes both high/intermediate-mass stars and lower-mass stars. Given the diversity in disk properties for 2.5 Myr-old IC 348, we suggest that a single flux slope may be best suited as a probe of disk evolution in the youngest regions (e.g. Taurus; NGC 1333), where less diversity in disk states (e.g. few if any debris disks) is expected.

\subsection{Constraints on the Primordial-to-Debris Disk Transition}

Recent Spitzer studies have placed strong constraints on the timescales for the evolution of primordial disks into debris disks. By $5 \mathrm{Myr}$, most high/intermediate-mass stars and solar-mass stars either lack evidence for a disk or have disk properties suggestive of a debris disk (Carpenter et al. 2006; Hernandez et al. 2008; Currie et al. 2009). At this age, many (detectable) subsolar-mass stars have weaker levels of mid-IR disk emission than typical primordial disks (Currie et al. 2009; Dahm and Carpenter 2009).

This work shows that by 2.5 Myr most disks around high/intermediate-mass stars are either actively leaving the primordial disk phase or have already reached the debris disk phase. Together with previous results (e.g. Carpenter et al. 2006; Hernandez et al. 2007; Currie et al. 2009), our results clearly support a stellar-mass dependent timescale for the disappearance of primordial disks and the emergence of debris disks. Within the context of planet formation, gas giant planets around high/intermediate-mass stars have much less time to form than around low-mass stars. If gas giant planets are more frequent around high/intermediate-mass stars (Johnson et al. 2007), their formation must be very rapid and efficient. Only recently have realistic models (Kenyon and Bromley 2009) that form gas giant planets via core accretion been successful in forming the cores of such planets in the short timescales implied from this paper $(2.5 \mathrm{Myr})$. Some trends in exoplanet properties, such as the semimajor axis distribution, may emerge from the competing effects of core formation timescales and primordial disk dispersal timescales (Currie 2009). 
The fraction of IC 348 disks in a transitional phase serves as a useful contrast to results obtained for younger clusters like Taurus and older clusters like NGC 2362. Based primarily on IRAS data, the computed frequency of transitional disks in Taurus is small, $<<10 \%$ (e.g. Skrutskie et al. 1990; Simon and Prato 1995; Wolk and Walter 1996). These authors then argued that the lifetime of transitional disks is $\approx 1-10 \%$ the age of Taurus: $\approx 0.01-0.1$ Myr. If this inference were correct, the total disk lifetime may be several Myr, but most of the disk is dissipated rapidly.

However, analysis of Spitzer data for 5 Myr-old NGC 2362 finds a much higher frequency of transitional disks (Currie et al. 2009). Both types of transitional disks (inner holes, homologously depleted) greatly outnumber primordial disks. This result argues for far longer typical transition timescale $(\approx 1 \mathrm{Myr})$.

In the intermediate age IC 348, the frequency of transition disks is intermediate between the frequency for 1 Myr-old Taurus and 5 Myr-old NGC 2362 for solar/subsolar-mass stars. Thus, the number of transition disks relative to primordial disks around solar/subsolar-mass stars appears to be an increasing function of stellar age. This result is expected if the typical transition timescale is an appreciable fraction of the typical primordial disk lifetime. Thus, the transition disks in Taurus and IC 348 could remain in such a state for an extended period. However, it is possible that transition disk lifetimes, like primordial disk lifetimes, also have an intrinsic dispersion (i.e., a gaussian distribution of lifetimes), where sources in Taurus represent those that make the primordial-to-debris disk transition fastest and sources in 5-10 Myr-old clusters make the transition the slowest. Quantifying the range of times a disk spends in a transitional phase and whether the typical timescale depends on stellar mass requires observations of many more 1-10 Myr-old clusters.

While debris disks typically only have excess emission longwards of $\approx 20 \mu \mathrm{m}$, the debris disk population in IC 348 contains at least two sources (ID-6 and ID-8) that have warmer dust more indicative of terrestrial planet formation than icy planet formation. Thus, warm debris disks consistent with the observable signatures of terrestrial planet formation may emerge as early as $\approx 2.5$ Myr around high/intermediate-mass stars. Observations of more 2-5 Myr-old clusters are needed to determine if the frequency of warm debris disks is higher than the $\sim$ 4\% derived for 10-15 Myr-old clusters (Currie et al. 2007a; Currie 2008). The existence of both warm debris disks and colder debris disks (lacking IRAC excess emission) suggests that even at 2.5 Myr debris disk populations may exhibit a range of dust temperatures consistent with a range of locations over which the debris-producing stages of planet formation are ongoing. 


\section{Acknowledgements}

We thank Taku Takeuchi for extensive discussions on the dynamics of circumstellar dust in optically-thin disks with and without circumstellar gas and for confirming the validity of the arguments presented in §3.4.3. We also thank Perry Berlind, Gautum Narayan, and Nathalie Martinbeau for scheduling, taking, and reducing spectra of IC 348 stars on short notice. Jesus Hernandez provided valuable discussions on Cycle 5 MIPS data for IC 348. Finally, we thank Charles Lada for informative discussions about previous Spitzer observations of IC 348. This work is supported by Spitzer GO grant 1320379, NASA Astrophysics Theory grant NAG5-13278, NASA grant NNG06GH25G, and Smithsonian Institution Restricted Endowment funds.

\section{REFERENCES}

Alexander, R., Clarke, C., Pringle, J., 2007, MNRAS, 369, 269

Allen, L., 1995, Ph.D. thesis, University of Massachusetts-Amherst

Andrews, S. M., Williams, J. P., 2005, ApJ, 631, 1134

Augereau, J., Lagrange, A. M., Mouillet, D., Papaloizou, J. C. B., Grorod, P. A., 1999, A\&A, 348, 557

Backman, D., Paresce, F., in Protostars and Planets III (E.H. Levy and J.I. Lunine, eds.) (Univ. of Arizona, Tucson), 1253, (1993)

Baraffe, I., Chabrier, G., Allard, F., Hauschildt, P. H., 1998, A\&A, 337, 403

Burns, J. A., Lamy, P. L., Soter, S., 1979, Icarus, 40, 1

Calvet, N., Hartmann, L., Kenyon, S. J., Whitney, B. A., 1994, ApJ, 434, 330

Carpenter, J., Mamajek, E. E., Hillenbrand, L. A., and Meyer, M., 2006, ApJ, 651, L49

Carpenter, J., et al., 2009, ApJS, 181, 197

Chen, C., Jura, M., Gordon, K. D., Blaylock, M., 2005, ApJ, 634, 1372

Currie, T., et al., 2007(a), ApJ, 659, 599

Currie, T., Kenyon, S. J., Rieke, G. H., Balog, Z., Bromley, B., 2007(b), ApJ, 663, L105

Currie, T., Kenyon, S. J., Balog, Z., Bragg, A., Tokarz, S., ApJ, 669, L33 
Currie, T., Kenyon, S. J., Balog, Z., Rieke, G. H., Bragg, A., and Bromley, B., 2008, ApJ, 672,558

Currie, T., 2008, Ph.D. thesis, University of California, Los Angeles

Currie, T., Plavchan, P., Kenyon, S. J., 2008, ApJ, 688, 597

Currie, T., Lada, C. J., Plavchan, P., Irwin, J., and Kenyon, S. J., 2009, ApJ, 698, 1

Currie, T., 2009, ApJ, 694, L171

D'Alessio, P., et al., 1999, ApJ, 527, 893

D’Antona, F., Mazzitelli, I., 1994, ApJS, 90, 467

Dahm, S., 2008, AJ, 136, 521

Dahm, S., Carpenter, J., 2009, AJ, 137, 4024

Ercolano, B., Clarke, C., Robitaille, T., 2009, MNRAS, 394, L141

Fabricant, D., et al., 1998, PASP, 110, 79

Flaherty, K., Muzerolle, J., 2007, ApJ, 663, 1069

Furlan, E., et al., 2006, ApJS, 165, 568

Gautier, T., Rebull, L., Stapelfeldt, K., Mainzer, A., 2008, ApJ, 683, 813

Hartmann, L., Calvet, N., Gullbring, E., D’Alessio, P., 1998, ApJ, 495, 385

Herbig, G., 1998, AJ, 497, 736

Hernandez, J., et al., 2007, ApJ, 662, 1067

Hernandez, J., et al., 2008, ApJ, 686, 1195

Hillenbrand, L., 2008, Physics Scripta, 130, 4024

Hillenbrand, L., et al., 2008, ApJ, 677, 630

Indebetouw, R., et al., 2005, ApJ, 619, 931

Isobe, T., Feigelson, E., ApJ, 306, 490

Johnson, J. A., et al., 2007, ApJ, 670, 833 
Kennedy, G., Kenyon, S. J., 2009, ApJ, 695, 1210

Kenyon, S., Bromley, B., 2004, ApJ, 602, 133L

Kenyon, S., Bromley, B., 2008, ApJS, 179, 451

Kenyon, S., Bromley, B., 2009, ApJ, 690, L140

Kenyon, S., Hartmann, L., 1995, ApJS, 101, 117

Kurucz, R. L., 1993, SYNTHE Synthesis Programs and Line Data, Kurucz CD-ROM No. 18, Cambridge, MA: Smithsonian Astrophysical Observatory, 1993, 18

Lada, C.J., et al., 2006, AJ, 131, 1574

LaValley, M., Isobe, T., and Feigelson, E., B.A.A.S., 1992, 22, 817

Lejeune, Th., Cuisinier, F., Buser, R., 1997, A\&AS, 125, 229

Lisse, C., Chen, C. H., Wyatt, M. C., Morlok, A., 2008, ApJ, 673, 1106

Low, F., Smith, P., Werner, M., Chen, C., Jura, M., 2005, ApJ, 631, 1170

Luhman, K., Rieke, G., Lada, C., Lada, E., 1998, ApJ, 508, 347

Luhman, K., 1999, ApJ, 525, 466

Luhman, K., Stauffer, J., Muench, A. A., Rieke, G. H., Lada, E. A., Bouvier, J., Lada, C. J., 2003, ApJ, 593, 1093

Luhman, K., Lada, E. A., Muench, A. A., Elston, R., 2005, ApJ, 618, 810

Makovoz, D., Khan, I., 2005, PASP, 347, 81

Makovoz, D., Marleau, F., 2005, PASP, 117, 1113

Mathis, J., 1990, ARA\&A, 28, 37

Mayne, N. J., Naylor, T., 2008, MNRAS, 386, 261

Muench, A., et al., 2003, AJ, 125, 2029

Muench, A., Lada, C. J., Luhman, K., Muzerolle, J., Young, E. T., 2007, AJ, 134, 411

Muzerolle, J., Calvet, N., Briceno, C., Hartmann, L., Hillenbrand, L., 2000, ApJ, 535, 47L

Muzerolle, J., Luhman, K., Briceno, C., Hartmann, L., Calvet, N., 2005, ApJ, 625, 906 
Muzerolle, J., et al., 2006, ApJ, 643, 1003

Plavchan, P., Jura, M., Lipscy, S., 2005, ApJ, 631, 1161

Plavchan, P., et al., 2009, ApJ, 698, 1068

Preibisch, T., Brown, A., Bridges, T., Guenther, E., Zinnecker, H., 2002, AJ, 124, 404

Preibisch, T., Zinnecker, H., 2001, AJ, 122, 866

Preibisch, T., Zinnecker, H., 2002, AJ, 123, 1613

Rebull, L., et al., 2008, ApJ, 681, 1484

Rhee, J., Song, I., and Zuckerman, B., 2007, ApJ, 671, 616

Rieke, G., et al., 2005, ApJ, 620, 1010

Robitaille, T. P., 2006, ApJS, 167, 256

Robitaille, T. P., 2007, ApJS, 169, 328 Shure, M., 1990, AJ, 99, 1187

Siess, L., Dufour, E., Forestini, M., 2000, A\&A, 358, 593

Simon, T., Prato, L., 1995, ApJ, 450, 824

Skrutskie, M., Dutkevitch, D., Strom, S., Edwards, S., Strom, K., 1990, AJ, 99, 1187

Strubbe, L., Chiang, E., 2006, ApJ, 648, 652

Su, K., et al., 2006, ApJ, 653, 675

Takeuchi, T., Artymowicz, P., 2001, ApJ, 557, 990

Walter, F., Vrba, F., Mathieu, R., Brown, A., Meyers, P., 1994, AJ, 107, 692

White, R. J., Basri, G., 2003, ApJ, 582, 1109

Wolk, S. J., Walter, F., 1996, AJ, 111, 2066 
Table 1. Spectroscopic data for Selected IC 348 sources

\begin{tabular}{|c|c|c|c|c|c|c|c|c|c|c|c|c|}
\hline ID Number & $\begin{array}{l}\text { Spectral } \\
\text { Type }\end{array}$ & $\begin{array}{l}\text { IRAC } \\
\text { Slope }\end{array}$ & SNR & $\begin{array}{l}\text { R EW } \\
\mathrm{H}_{\alpha}(\AA)\end{array}$ & Emission? & $\begin{array}{l}\text { FWHM } \\
\mathrm{H}_{\alpha}(\AA)\end{array}$ & $\begin{array}{l}\text { EW H }{ }_{\alpha} \\
\operatorname{Em} .(\AA)\end{array}$ & $\mathrm{R}_{c}$ (est.) & $\begin{array}{l}\mathrm{R}_{\star} \\
\left(\mathrm{R}_{\odot}\right)\end{array}$ & $\begin{array}{l}\mathrm{M}_{\star} \\
\left(\mathrm{M}_{\odot}\right)\end{array}$ & $\begin{array}{l}\text { Flux }\left(\mathrm{H}_{\alpha}\right) \\
\left(\mathrm{W} \mathrm{m} \mathrm{m}^{-2}\right)\end{array}$ & $\dot{M} / \dot{M}_{I D-21}$ \\
\hline 2 & $\mathrm{~A} 2$ & thick & 143 & -10.7 & maybe & - & $<0.015$ & 7.2 & 4.5 & 3.1 & $<3.8 \times 10^{-17}$ & $<0.07$ \\
\hline 3 & A0 & diskless & 170 & -6.3 & no & - & $<0.02$ & 7.3 & 4.0 & 3.0 & $<4.6 \times 10^{-17}$ & $<0.08$ \\
\hline 6 & G3 & anemic & 105 & -1.0 & probably not & - & $<0.1$ & 9.0 & 3.9 & 2.3 & $<4.8 \times 10^{-17}$ & $<0.10$ \\
\hline 8 & $\mathrm{~A} 2$ & anemic & 156 & -10.9 & no & - & $<0.02$ & 8.51 & 2.4 & 2.3 & $<1.5 \times 10^{-17}$ & $<0.02$ \\
\hline 19 & $\mathrm{~A} 2$ & thick & 132 & -10.0 & yes & 2.9 & 0.64 & 9.15 & 1.7 & 2.1 & $2.6 \times 10^{-16}$ & 0.3 \\
\hline 21 & K0 & anemic & 80 & 5.0 & yes & 7.8 & 5.0 & 10.48 & 2.5 & 1.8 & $6 \times 10^{-16}$ & 1 \\
\hline 30 & F0 & diskless & 123 & -6.2 & no & - & $<0.02$ & 10.06 & 1.6 & 1.6 & $<3.5 \times 10^{-18}$ & $<0.004$ \\
\hline
\end{tabular}

Note. - Negative $\mathrm{EW}\left(\mathrm{H}_{\alpha}\right)$ indicate absorption and positive values indicate emission. IC348 ID-19 has a central emission core in the deep absorption line. The $\mathrm{H}_{\alpha}$ line for ID-6 is weak for G3 stars, though there is no clear central emission feature and the star is very $\mathrm{x}$-ray luminous.

Table 2. IC 348 Stars with New MIPS $24 \mu m$ Detections

\begin{tabular}{|c|c|c|c|c|c|c|}
\hline ID & RA & DEC & Offset(") & Spectral Type & IRAC Slope [24] & $\sigma[24] \mathrm{K}_{s^{-}}[24]$ (dereddened) \\
\hline 4 & 56.1300 & 32.1061 & 1.80 & F0 & 6.402 & 0.0971 .24 \\
\hline 39 & 56.2573 & 32.2411 & 13.68 & K4 & 8.818 & 0.2250 .87 \\
\hline 47 & 55.9813 & 32.1590 & 1.43 & K0 & 10.488 & $0.280-0.42$ \\
\hline 49 & 55.9900 & 32.0271 & 3.72 & M0.5 & 8.076 & 0.4300 .53 \\
\hline 53 & 56.0684 & 32.1653 & 30.29 & K0 & 9.545 & 0.2570 .62 \\
\hline 56 & 56.0208 & 32.1649 & 1.62 & K3.5 & 9.838 & 0.1950 .45 \\
\hline 83 & 56.1559 & 32.1503 & 30.18 & M1 & 5.733 & 0.2865 .05 \\
\hline 85 & 56.1172 & 32.2668 & 30.45 & M3.25 & 9.078 & 0.3501 .84 \\
\hline 88 & 56.1365 & 32.1544 & 42.97 & M3.25 & 6.819 & 0.4254 .00 \\
\hline 91 & 56.1634 & 32.1624 & 41.90 & M2 & 8.264 & 0.2652 .63 \\
\hline 95 & 56.0913 & 32.2032 & 21.16 & M4 & 9.118 & 0.3111 .88 \\
\hline 124 & 55.9776 & 32.0084 & $4-$ & M4.25 & 9.144 & 0.1902 .19 \\
\hline 167 & 56.1716 & 32.1695 & 0.47 & M3 & 7.246 & 0.5653 .93 \\
\hline 168 & 56.1306 & 32.1797 & 71.15 & M4.25 & 7.145 & 0.2634 .37 \\
\hline 193 & 56.1584 & 32.1936 & 0.85 & M4 & 7.141 & 0.1824 .76 \\
\hline 261 & 55.9526 & 32.2308 & 31.96 & M5 & 10.037 & 0.2032 .65 \\
\hline 300 & 56.1624 & 32.0555 & 51.49 & M5 & 7.735 & 0.2655 .23 \\
\hline 336 & 56.1349 & 32.0576 & 0.95 & M5.5 & 8.058 & 0.3355 .27 \\
\hline 341 & 56.0541 & 32.2210 & 1.08 & M5.25 & 8.853 & 0.3044 .28 \\
\hline 365 & 56.0426 & 32.1263 & 1.26 & M5.75 & 9.383 & 0.3404 .01 \\
\hline 373 & 56.1166 & 32.0888 & $3-$ & M5.5 & 8.418 & 0.1075 .31 \\
\hline 402 & 56.1898 & 32.3055 & 50.41 & M5.5 & 8.611 & 0.1744 .92 \\
\hline 407 & 56.2672 & 32.0846 & 0.25 & M7 & 9.578 & 0.1644 .89 \\
\hline 746 & 56.2082 & 32.1041 & 1.40 & M5 & 7.683 & $0.260 ?$ \\
\hline 1124 & 56.2364 & 32.2844 & 40.86 & M5 & 8.406 & 0.1983 .53 \\
\hline 1939 & 56.2198 & 32.0158 & 31.11 & M4.75 & 10.584 & 0.3130 .82 \\
\hline 2096 & 56.0539 & 32.2234 & 40.39 & M6 & 8.713 & $0.233 ?$ \\
\hline
\end{tabular}

Note. - The IRAC slope follows the convention of Lada et al. (2006): 1 = 'thick', 2 = 'anemic', and $3=$ 'diskless'. The equivalent designations in Currie et al. (2009) are the following: $1=$ 'strong IRAC', 2 = 'weak IRAC', and $3=$ 'photosphere'. 
Table 3. IC 348 Stars With MIPS $70 \mu m$ Detections

\begin{tabular}{llllll}
\hline \hline \multicolumn{1}{l}{ IC348-ID Spectral Type Source Type } & $\mathrm{F}_{70}(\mathrm{mJy})$ & $\sigma\left(\mathrm{F}_{70}\right)$ & $(\mathrm{mJy})$ & Offset(") \\
\hline 13 & M0.5 & 1 & 1408 & 103.7 & 2.85 \\
31 & G1 & 2 & 556.2 & 140.3 & 2.86 \\
51 & - & 1 & 3972.0 & 127.0 & 1.74 \\
67 & M0.75 & 2 & 125.5 & 18.4 & 2.08 \\
10343 & M3.75 & 1 & 115.6 & 15.9 & 1.96 \\
10352 & M1 & 1 & 322.1 & 25.1 & 2.37 \\
\hline
\end{tabular}

Table 4. Catalog of IC 348 Stars

\begin{tabular}{lllllllllllllllllllllllll}
\hline \hline ID RA & \multirow{2}{*}{$\mathrm{DEC}$} & $\mathrm{J}$ & $\mathrm{H}$ & $\mathrm{K}_{s}$ & {$[3.6]$} & $\sigma[3.6]$ & {$[4.5]$} & $\sigma[4.5]$ & {$[5.8]$} & $\sigma[5.8]$ & {$[8]$} & $\sigma[8]$ & {$[24]$} & $\sigma[24]$ & {$[70]$} & $\sigma[70]$ & $\mathrm{ST}$ & $\mathrm{A}_{V}$ & $\mathrm{IRA}$ \\
\hline 1 & 56.1425 & 32.1630 & 6.790 & 6.640 & 6.510 & 6.740 & 0.010 & 6.540 & 0.020 & 6.580 & 0.020 & 6.500 & 0.030 & 1.874 & -9 & -2.267 & -9 & 15 & 3.10 & 3 \\
2 & 56.1474 & 32.1679 & 7.950 & 7.530 & 7.250 & 7.090 & 0.020 & 6.810 & 0.020 & 6.460 & 0.040 & 5.820 & 0.040 & 3.375 & 0.038 & -2.176 & -9 & 22 & 3.20 & 1 \\
3 & 56.2110 & 32.3185 & 8.370 & 7.900 & 7.660 & 7.530 & 0.010 & 7.610 & 0.030 & 7.470 & 0.040 & 7.500 & 0.040 & 7.086 & 0.141 & -1.009 & -9 & 20 & 3.90 & 3 \\
4 & 56.1300 & 32.1061 & 8.340 & 8.020 & 7.860 & 7.790 & 0.010 & 7.740 & 0.020 & 7.660 & 0.020 & 7.730 & 0.030 & 6.402 & 0.097 & -0.898 & -9 & 30 & 2.30 & 3 \\
5 & 56.1084 & 32.0751 & 10.070 & 8.870 & 8.140 & 6.970 & 0.010 & 6.520 & 0.010 & 6.320 & 0.020 & 5.630 & 0.020 & 2.655 & 0.012 & -0.699 & -9 & 48 & 7.70 & 1 \\
\hline
\end{tabular}

Note. - Full catalog of IC 348 sources from Lada et al. (2006) including MIPS photometry for newly-detected stars from this work. The column labeled "ST" indicates the numerical spectral type (e.g., 15=B5, 22=A2). 
Table 5. Evolutionary State of Disks with New $24 \mu m$ Detections

\begin{tabular}{|c|c|c|c|c|c|}
\hline ID & Spectral & Type IRAC Slope & $\mathrm{K}_{s^{-}}[8]$ (dereddened) & $\mathrm{K}_{s^{-}}[24]$ (dereddened) & Disk State \\
\hline 4 & F0 & 3 & -0.01 & 1.24 & 4 \\
\hline 39 & $\mathrm{~K} 4$ & 3 & 0.08 & 0.87 & 4 \\
\hline 47 & K0 & 3 & 0.10 & -0.42 & 5 \\
\hline 49 & M0.5 & 3 & 0.24 & 0.53 & 5 \\
\hline 53 & K0 & 3 & 0.01 & 0.62 & 4 \\
\hline 56 & K 3.5 & 3 & 0.02 & 0.45 & 4 \\
\hline 83 & M1 & 1 & 2.00 & 5.05 & 1 \\
\hline 85 & M3.25 & 3 & 0.26 & 1.84 & 2 \\
\hline 88 & M3.25 & 2 & 0.60 & 4.00 & 3 \\
\hline 91 & M2 & 1 & 1.71 & 2.63 & 2 \\
\hline 95 & M4 & 3 & 0.39 & 1.88 & 2 \\
\hline 124 & M4.25 & 3 & 0.55 & 2.19 & 2 \\
\hline 167 & M3 & 2 & 1.16 & 3.93 & 2 \\
\hline 168 & M4.25 & 1 & 1.91 & 4.37 & 1 \\
\hline 193 & M4 & 1 & 1.76 & 4.76 & 1 \\
\hline 261 & M5 & 2 & 0.66 & 2.65 & 2 \\
\hline 300 & M5 & 1 & 1.97 & 5.23 & 1 \\
\hline 336 & M5.5 & 1 & 1.99 & 5.27 & 1 \\
\hline 341 & M5.25 & 1 & 1.40 & 4.28 & 1 \\
\hline 365 & M5.75 & 2 & 1.55 & 4.01 & 1 \\
\hline 373 & M5.5 & 2 & - & 5.31 & 3 \\
\hline 402 & M5.5 & 2 & 1.32 & 4.92 & 1 \\
\hline 407 & M7 & 1 & 2.15 & 4.89 & 1 \\
\hline 746 & M5 & 1 & - & - & - \\
\hline 1124 & M5 & 2 & 0.70 & 3.53 & 2 \\
\hline 1939 & M4.75 & 3 & 0.39 & 0.82 & 4 \\
\hline 2096 & M6 & 1 & - & - & - \\
\hline
\end{tabular}

Note. - The IRAC slope follows the convention of Lada et al. (2006): $1=$ 'thick', $2=$ 'anemic', and $3=$ 'diskless'. The disk states are $1=$ primordial, $2=$ homologously depleted, $3=$ disks with inner holes, $4=$ debris disk candidates, $5=$ photospheres (no disk).

Table 6. Dust Removal Timescales and Dust Masses for Representative Debris Disk Candidates

\begin{tabular}{|c|c|c|c|c|c|c|c|c|}
\hline ID & $\begin{array}{l}\text { IRAC }^{a} \\
\text { Slope }\end{array}$ & $\begin{array}{l}\text { Spectr } \\
\text { Type }\end{array}$ & $1 \log \left(L_{\star}\right.$ & $\mathrm{M} / \mathrm{M}_{\odot}$ & $\begin{array}{l}\mathrm{t}_{p r}(\mathrm{Myr}) \\
(1,10,30 \mathrm{AU})\end{array}$ & $\begin{array}{l}\mathrm{s}_{\max } \\
(\mu m)\end{array}$ & $\begin{array}{l}\text { Disk }^{b} \\
\text { Model }\end{array}$ & $\begin{array}{r}\mathrm{M}_{d u} \\
(\times 10\end{array}$ \\
\hline 6 & Anemic & G3 & 1.209 & 2.6 & $4.3 \times 10^{-5}, 4.3 \times 10^{-3}, 0.039$ & 7.1 & $3006897-5-9$ & 8.77 \\
\hline 8 & Anemic & $\mathrm{A} 2$ & 1.509 & 2.8 & $2.2 \times 10^{-5}, 2.2 \times 10^{-3}, 0.020$ & 13.1 & $3004289-5-9$ & 2.48 \\
\hline 20 & Diskless & G1 & 0.719 & 2.6 & $1.3 \times 10^{-4}, 0.013,0.120$ & 2.3 & $3001439-5-9$ & 2.09 \\
\hline 30 & Diskless & & 0.806 & 2.6 & $1.1 \times 10^{-4}, 0.011,0.098$ & 2.8 & $3003786-5-9$ & 2.95 \\
\hline 39 & Diskless & $\mathrm{K} 4$ & 0.229 & 1.3 & $4.1 \times 10^{-4}, 0.041,0.371$ & 1.5 & $3008796-8-9$ & 20.0 \\
\hline 1939 & Diskless & M4 4.75 & -0.792 & 0.2 & $4.4 \times 10^{-3}, 0.44,3.9$ & 0.98 & $3002293-6-9$ & 14.0 \\
\hline
\end{tabular}

Note. - Constraints on the dust removal timescales and disk masses for debris disk candidates. The Poynting-Robertson drag timescale is calculated assuming $1 \mathrm{\mu m}$-sized grains. Disk models listed represent the file number from the Robitaille et al. (2006) grid; dust masses for those models are listed as $\mathrm{M}_{d u s t}$. a) From Lada et al. (2006). b) From Robitaille et al. (2006). 

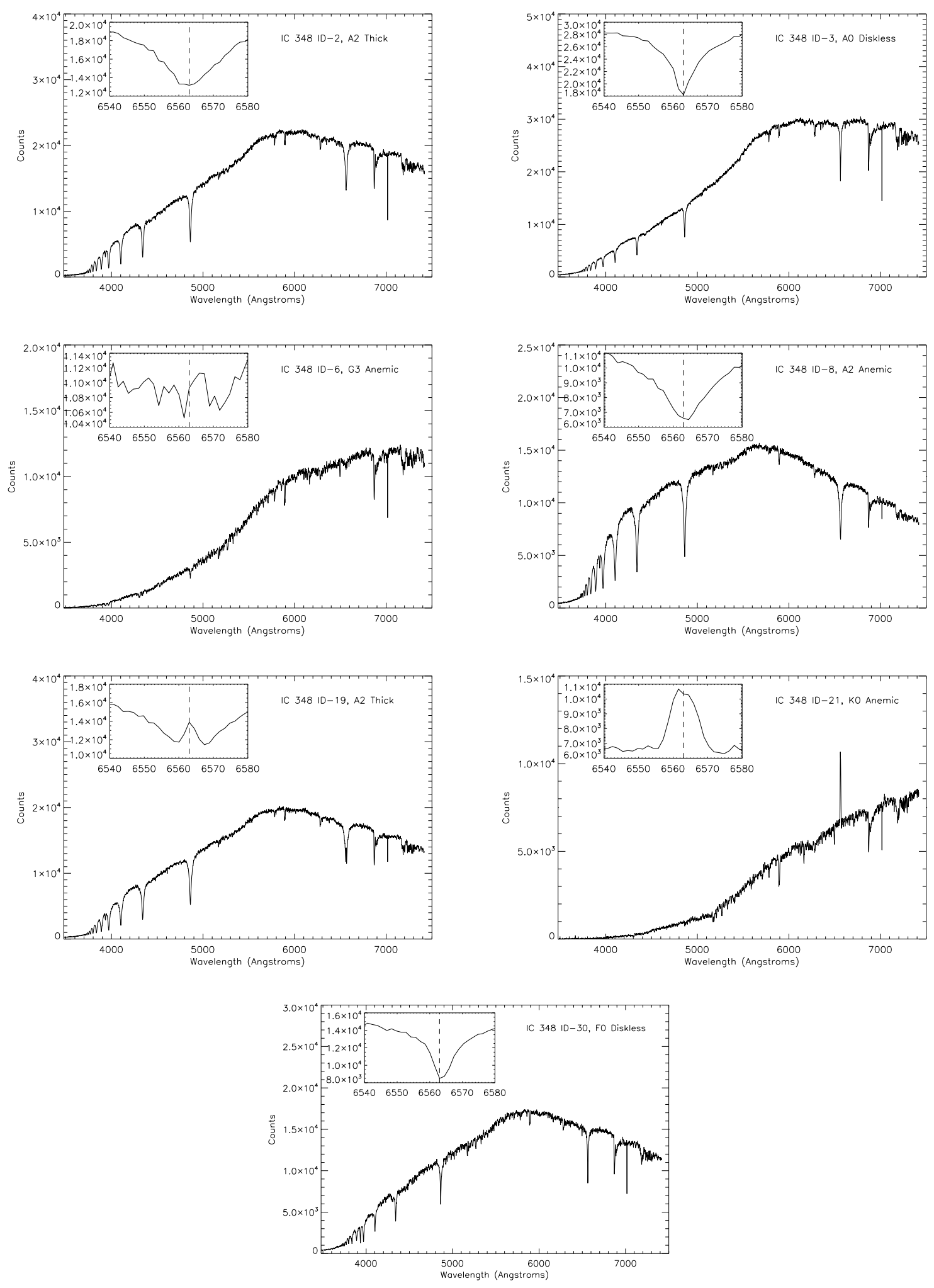

Fig. 1. - FAST spectra of IC 348 sources. The spectra have not been smoothed. Each source is labled by its catalog number from Lada et al. (2006), its spectral type, and its IRAC slope. ID-21 (K0 anemic) has a strong, wide $\mathrm{H}_{\alpha}$ emission line; ID-19 (A2 thick) also has a $\mathrm{H}_{\alpha}$ emission core in its absorption line. ID-6 (G3 anemic) has $\mathrm{H}_{\alpha}$ nearly filled in, 

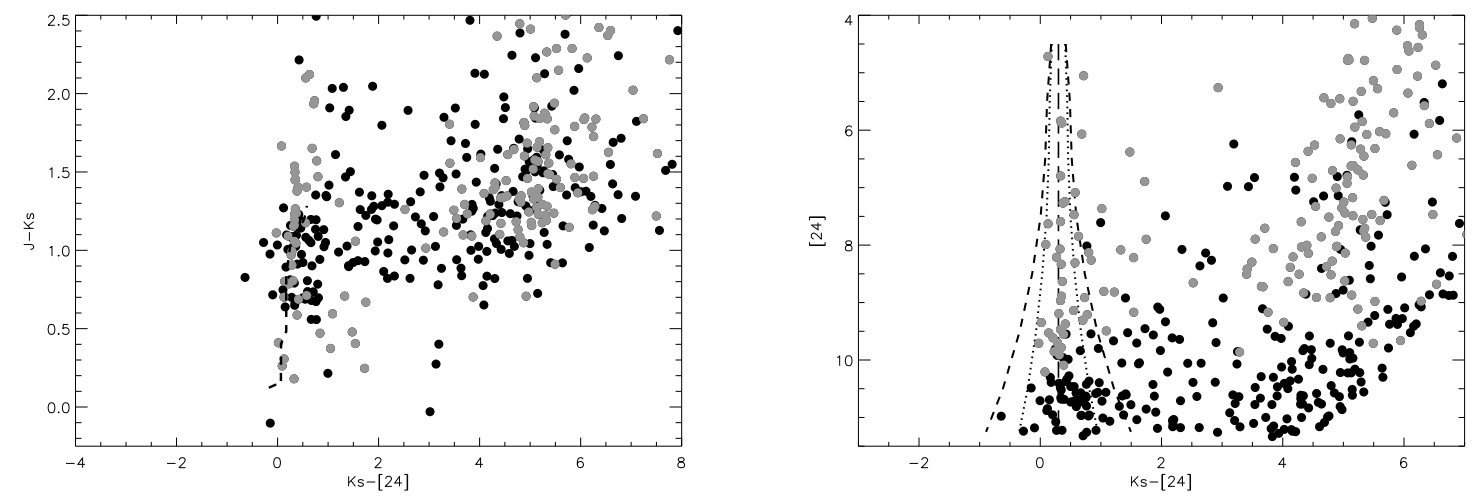

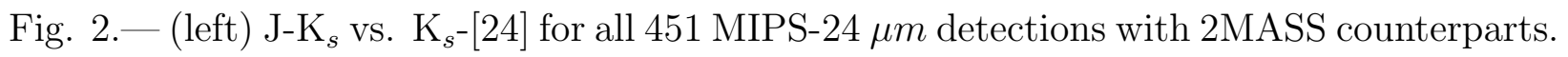
The dashed line identifies the locus of photospheric colors for a star reddened by $\mathrm{A}_{V}=2$. (right) The [24] vs. $\mathrm{K}_{s^{-}}[24]$ color-magnitude diagram for the same sources. The long dashed line identifies the photospheric locus for a star with $\mathrm{K}_{s^{-}}[24]=0.25$ : a mid-K star reddened by $\mathrm{A}_{V}=2$ or an unreddened M2-M3 star. The dotted line, short-dashed line, and dash-three dots line correspond to the 1 and $2 \sigma$ limits from the combined $K_{s}$ and [24] photometric uncertainties assuming $\mathrm{a} \sim 1$ magnitude spread in extinction. 


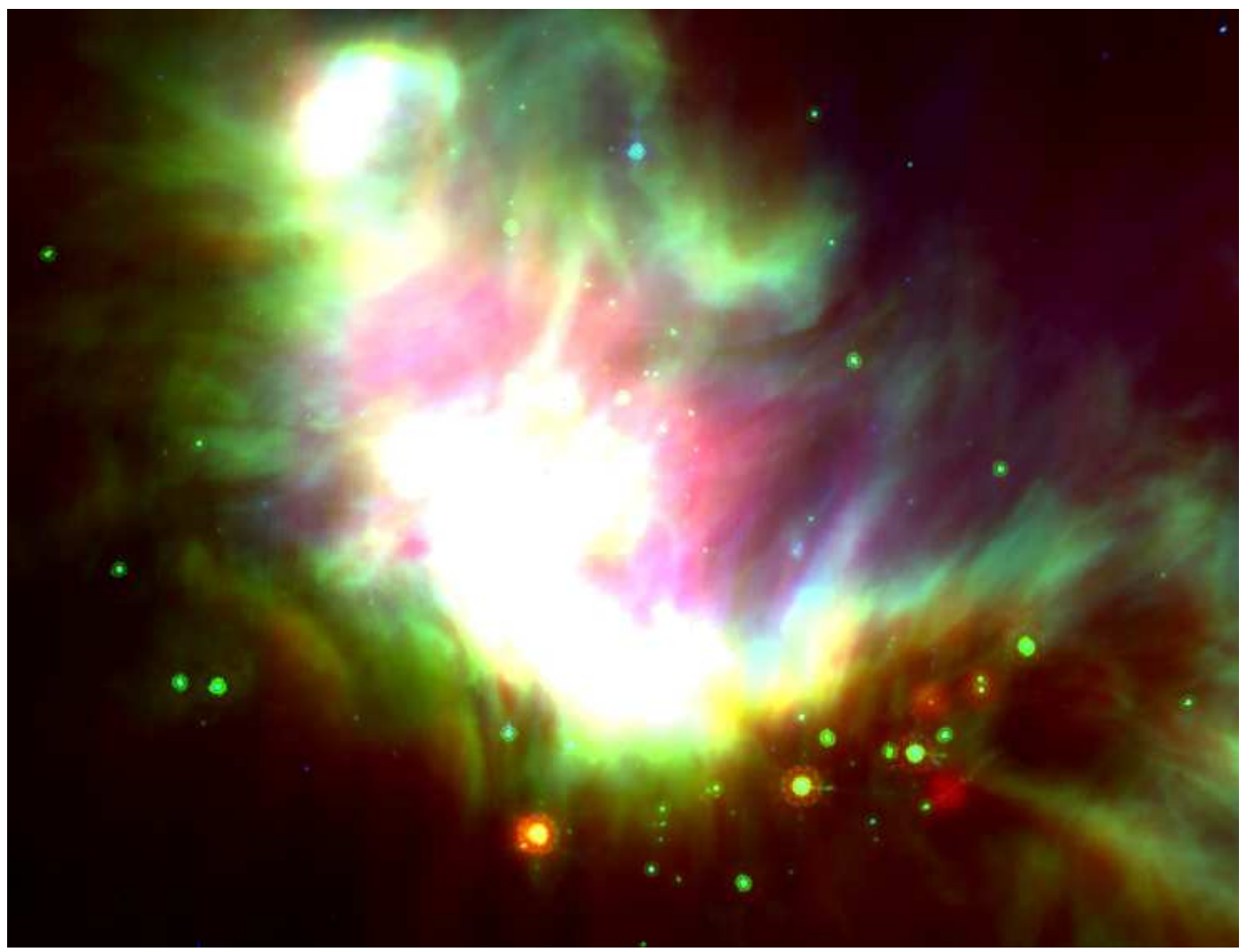

Fig. 3.- Three color mosaic of the IC 348 Nebula. Blue represents IRAC $8 \mu m$ Cycle 1 data produced by the post-bcd pipeline mosaic; green and red colors represent the MIPS-24 $\mu \mathrm{m}$ and $70 \mu \mathrm{m}$ emission from the processed data described in this paper. 

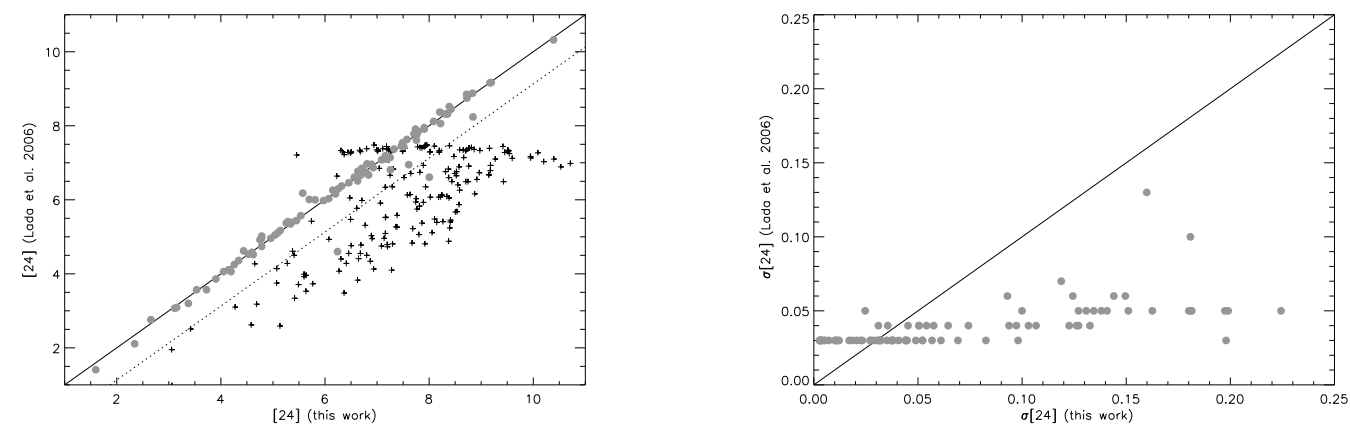

Fig. 4.- Comparisons of our MIPS $24 \mu \mathrm{m}$ photometry/upper limits (left) and photometric errors (right) with those from Lada et al. (2006). In the left panel, grey dots identify magnitudes for sources detected from both datasets and crosses identify the $2 \sigma$ upper limits for sources that lack detections in either dataset. The solid line identifies where our [24] photometry and that from Lada et al. (2006) would be in perfect agreement in the photon-noise limit. The dotted line represents the expected locus of upper limits, given our exposure time, based on the Lada et al. (2006) upper limits. In the right panel, grey dots represent photometric errors and the solid line identifies where our photometric errors are identical.
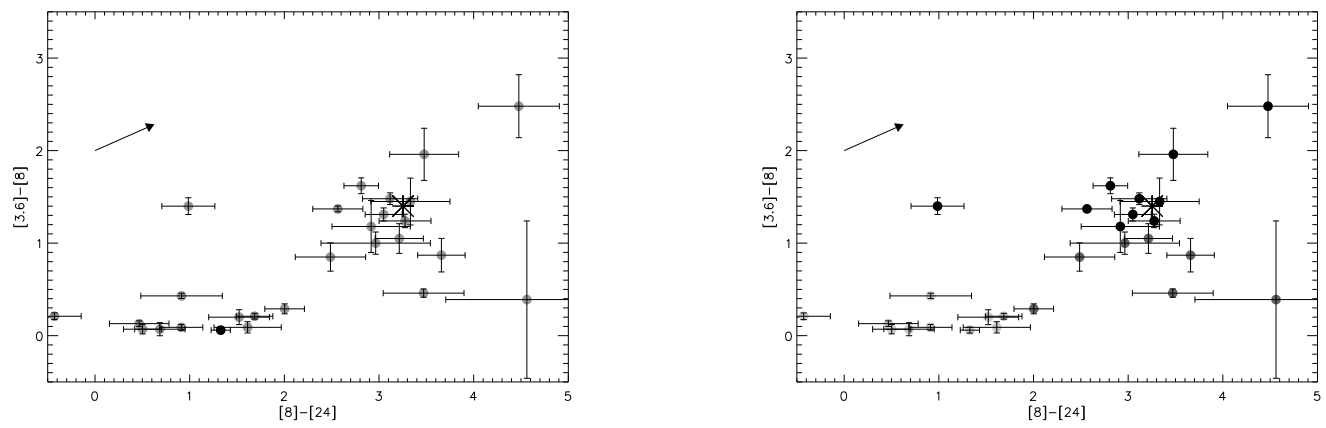

Fig. 5.- [3.6]-[8] vs. [8]-[24] color-color diagram for IC 348 stars with new MIPS $24 \mu \mathrm{m}$ detections. Overplotted are the $1 \sigma$ error bars. For comparison, we show the colors for the median Taurus SED (asterisks). (Left) Stars with spectral types earlier than K0 are shown as dark circles; stars with spectral types later than K0 are shown as grey circles. (Right) Stars identified as having strong ('thick') IRAC excess emission are dark circles, stars with weak ('anemic') IRAC excess emission are grey, and stars lacking IRAC excess emission ('diskless') are light grey. In both plots, a reddening vector of $\mathrm{A}_{v}=20$ is overplotted, where we assume extinction laws from Indebetouw et al. (2005) for IRAC and approximate the 24 $\mu m$ extinction from Mathis (1990). We note that Flaherty and Muzerolle (2007) estimate that the extinction law is much flatter from $8 \mu m$ to $24 \mu m$ than assumed here. 


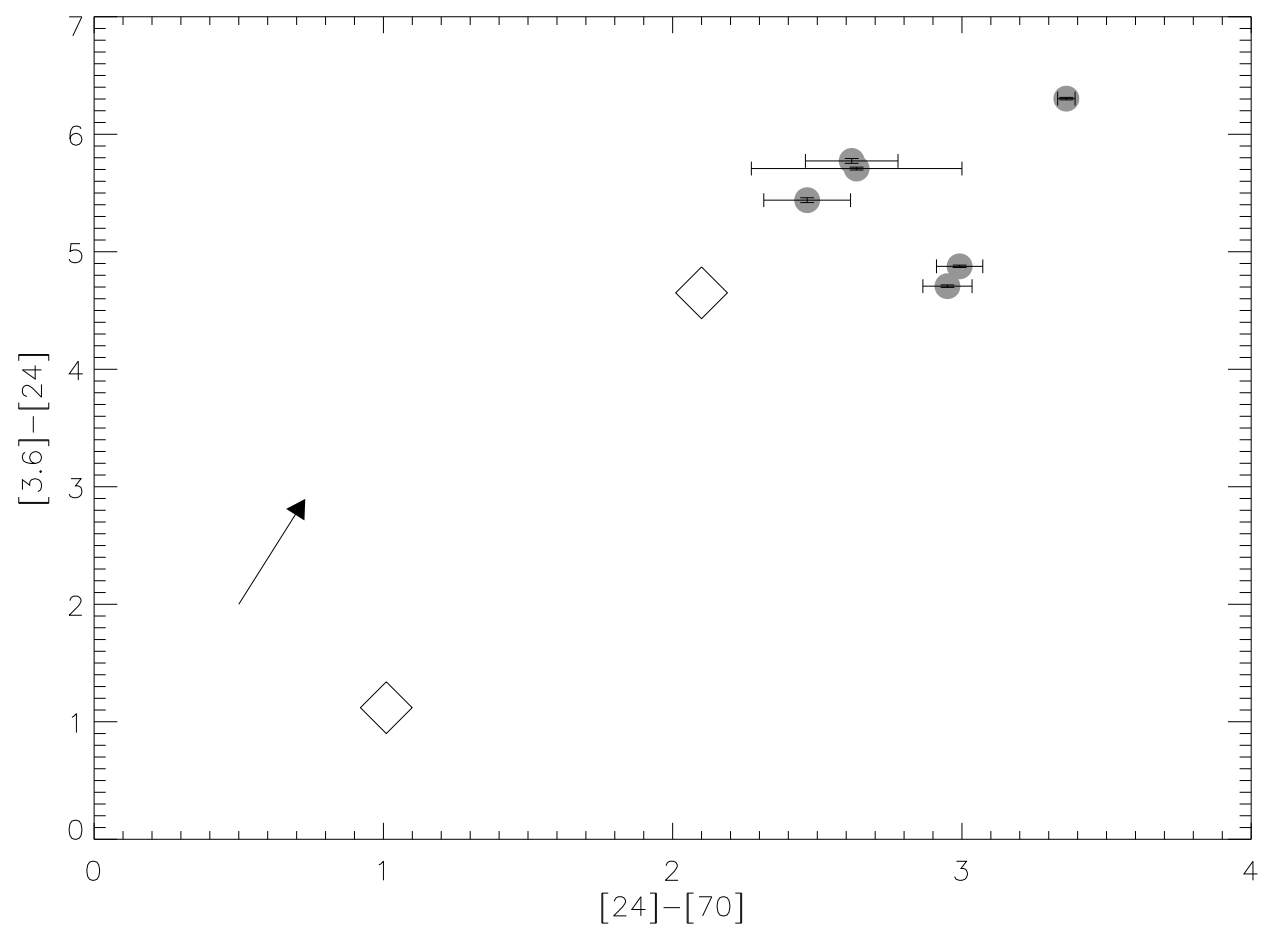

Fig. 6.- [3.6]-[24] vs. [24]-[70] color-color diagram for IC 348 stars detected at $70 \mu m$ (dots). Overplotted are the positions of two $\eta$ Cha stars (diamonds), ECH-2 (lower left) and ECH-15 (upper right). 

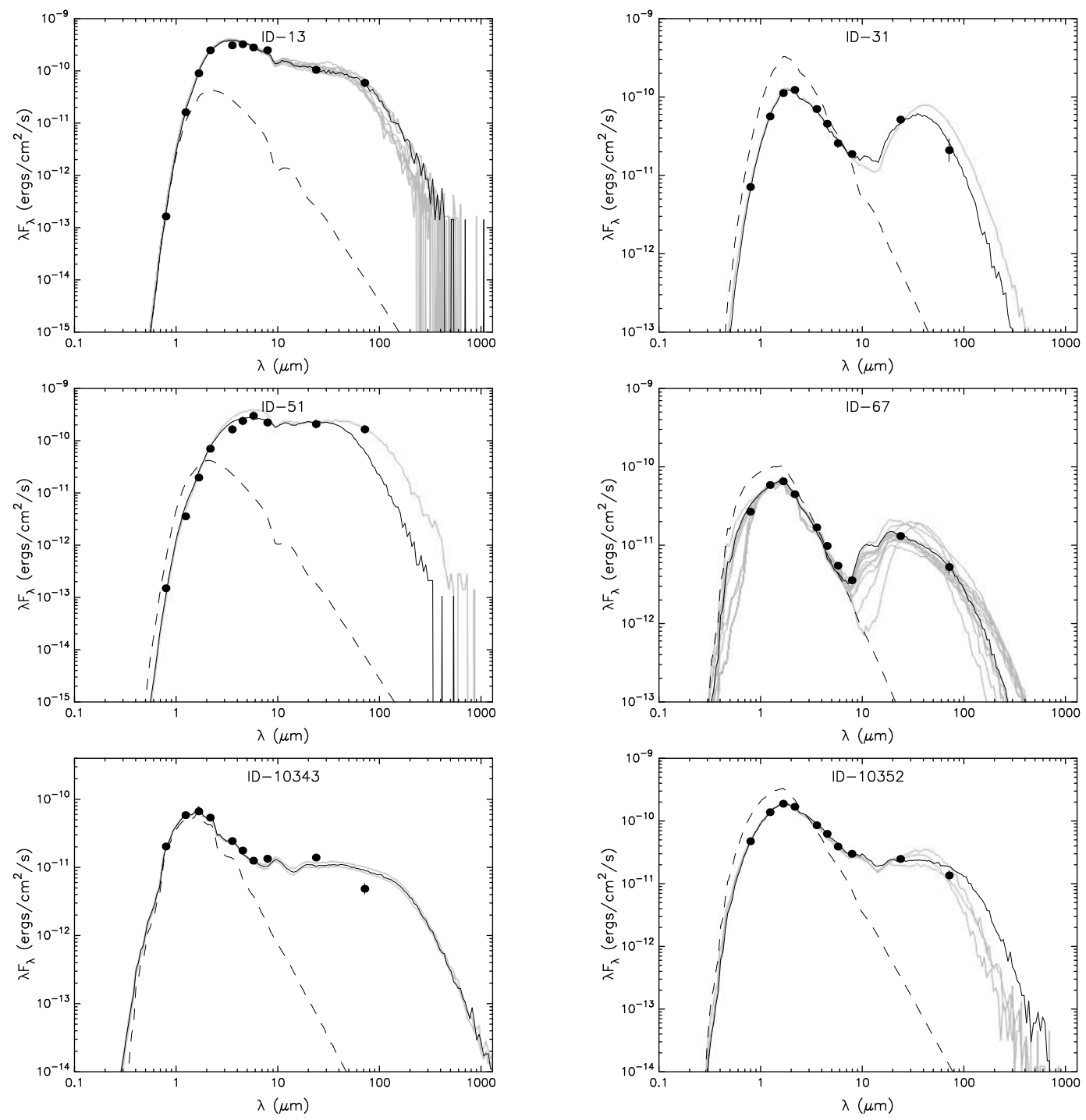

Fig. 7.- SED fits to IC 348 sources detected at $70 \mu \mathrm{m}$ using the Robitaille et al. (2006) models. The dotted line corresponds to the unreddened SED of the best-fit stellar photosphere. The solid black line corresponds to the best-fit SED Robitaille et al. (2006) model. The light grey lines correspond to other Robitaille et al. (2006) models with a $\chi^{2}-\chi_{\text {best }}^{2}<10$. 

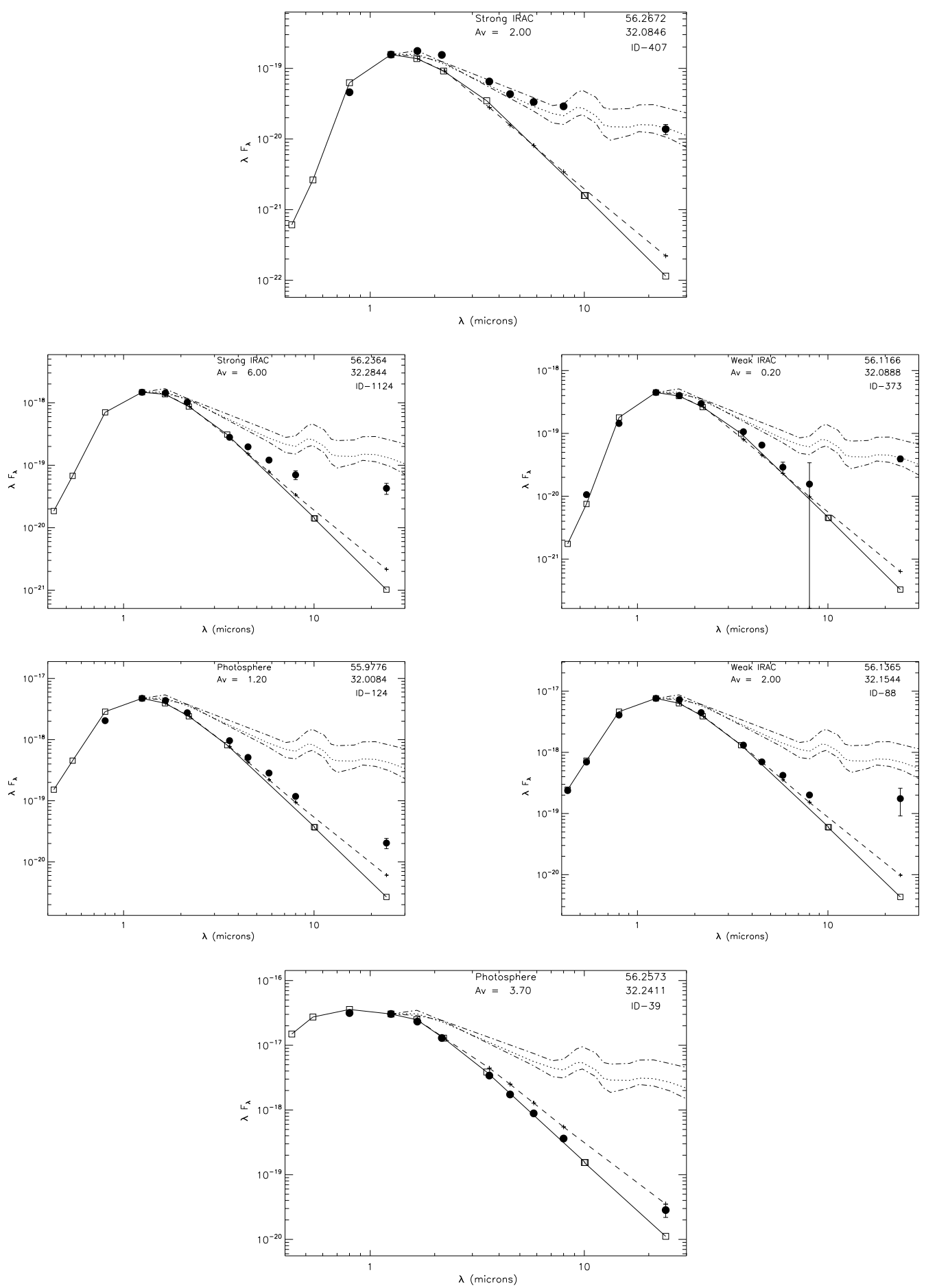

Fig. 8.- Representative SEDs of IC 348 stars with new MIPS $24 \mu m$ detections. Data points are black dots with photometric errors overplotted. The solid line corresponds to a stellar photosphere appropriate for the star's spectral type, the dashed line corresponds to the debris disk model from Kenyon and Bromley (2004), the dotted line corresponds to the SED of the median Taurus SED, and the dot-dashed lines correspond to the upper and lower quartiles for the median Taurus SED. Each source is identified by its IRAC slope (Strong IRAC $=$ 'thick', Weak IRAC = 'anemic', Photosphere='diskless), its optical extinction, its J2000 position in decimal degrees, and its catalog ID number. 

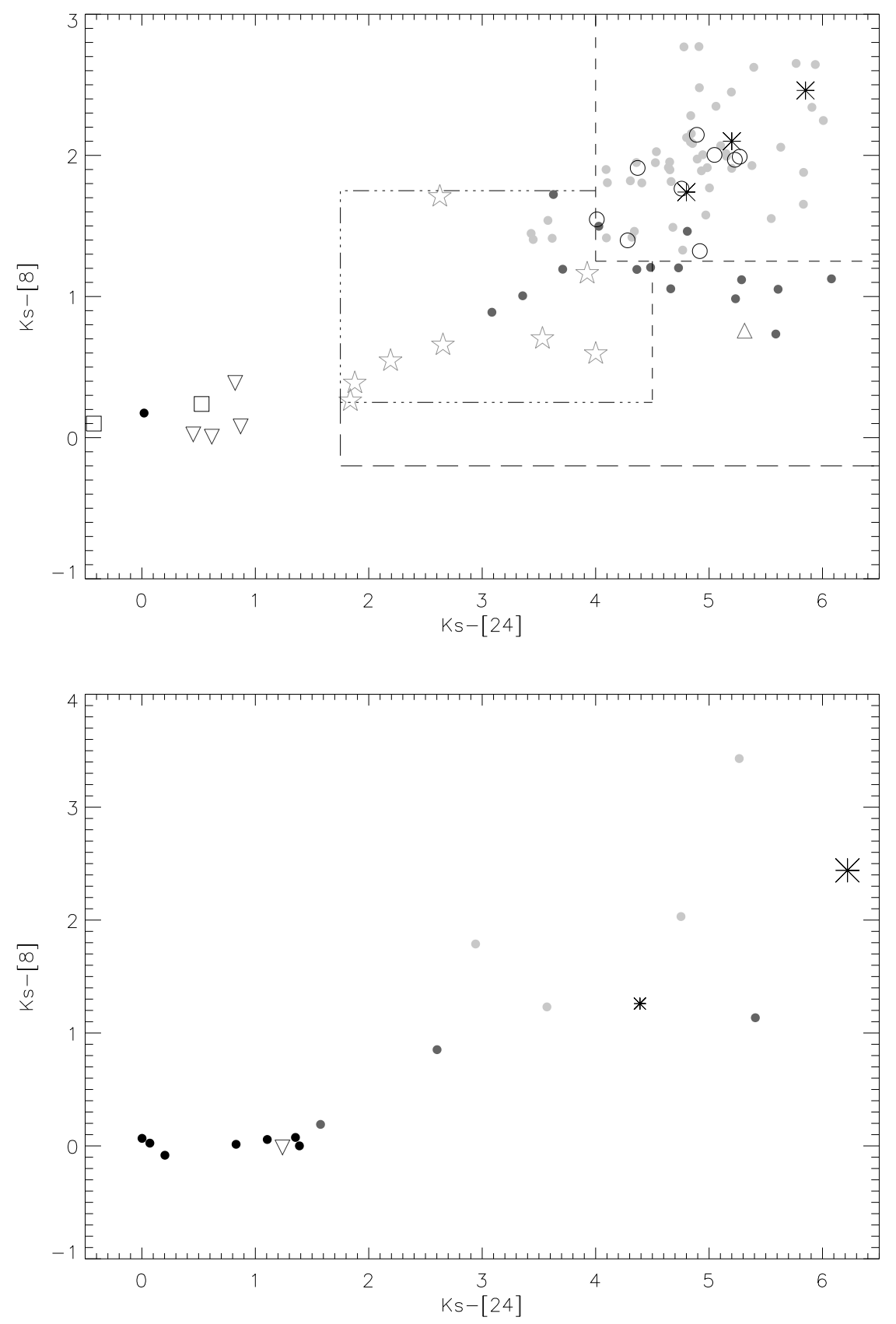

Fig. 9.- (top) $\mathrm{K}_{s^{-}}[8]$ vs. $\mathrm{K}_{s^{-}}[24]$ color-color diagram for sources having spectral types $\mathrm{K} 0$ and later with new MIPS detections (open symbols) and previously-detected sources (filled dots) later than K0. The colors are dereddened using $A_{V}$ values from Muench et al. (2007). For sources with new detections, squares correspond to bare photospheres, inverted triangles are debris disk candidates, triangles are disks with inner holes, stars are homologously depleted disks, and open circles are primordial disks. The disk population is well separated in colorcolor space (dashed and dash-dotted lines). Previously detected sources labeled as 'thick' tend to reside in the primordial disk region, sources identified as anemic are located in the homologously depleted and inner-holed regions, and 'diskless' sources are located in the debris disk/photospheric region. (bottom) The same diagram for sources with spectral types 

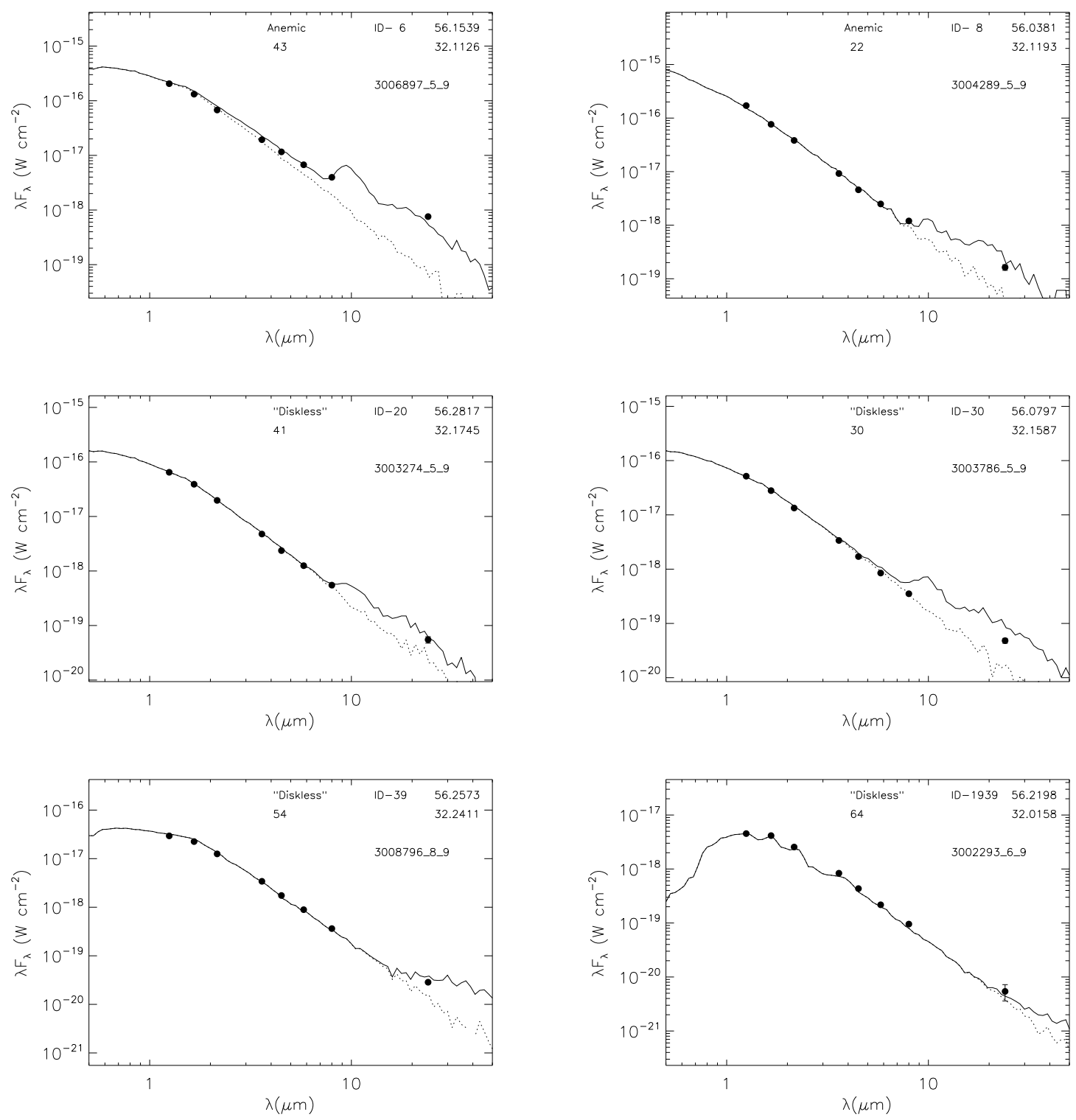

Fig. 10. - SED of representative debris disk candidates. Overplotted are the best-fit SEDs from Robitaille et al. (2006, solid line) along with the stellar photosphere(dotted line). Also listed are the Lada et al. (2006) IRAC slope and ID number, the numerical spectral type (below the IRAC slope), the J2000 source position (in degrees), and the model number for the best-fit Robitaille et al. (2006) model SED. Photometric uncertainties $(1 \sigma)$ are typically smaller than the symbol sizes. 

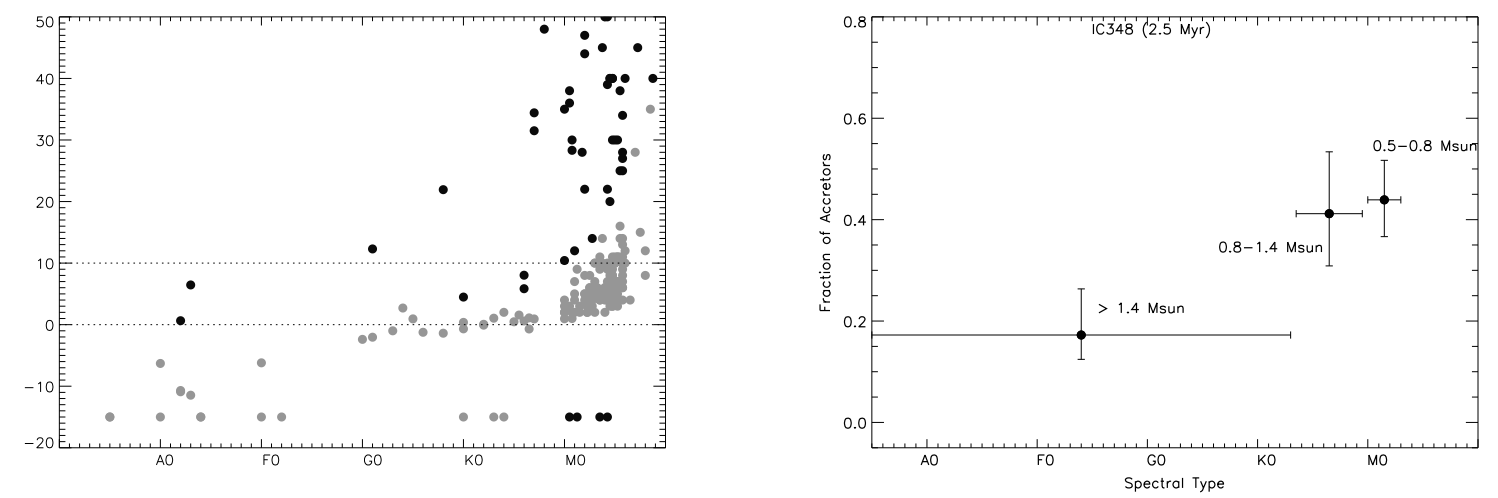

Fig. 11. - (left) $\mathrm{H}_{\alpha}$ equivalent width versus spectral type for IC 348 stars. Accretors are shown as black dots; nonaccretors are grey dots. Sources without measured $\mathrm{EW}\left(\mathrm{H}_{\alpha}\right)$ values have $\operatorname{EW}\left(\mathrm{H}_{\alpha}\right)$ set to $-15 \AA$. For reference, we draw a line corresponding to the division between Classical T Tauri stars and Weak-line T Tauri stars. (right) Frequency of accretors as a function of spectral type for three spectral type bins. The horizontal error bars correspond to the bin size in spectral type - B5-K3, K3-M0, and M0-M2.5 - and the vertical error bar identifies the $1 \sigma$ uncertainty in the frequency of accretors for each spectral type bin based on binomial statistics.
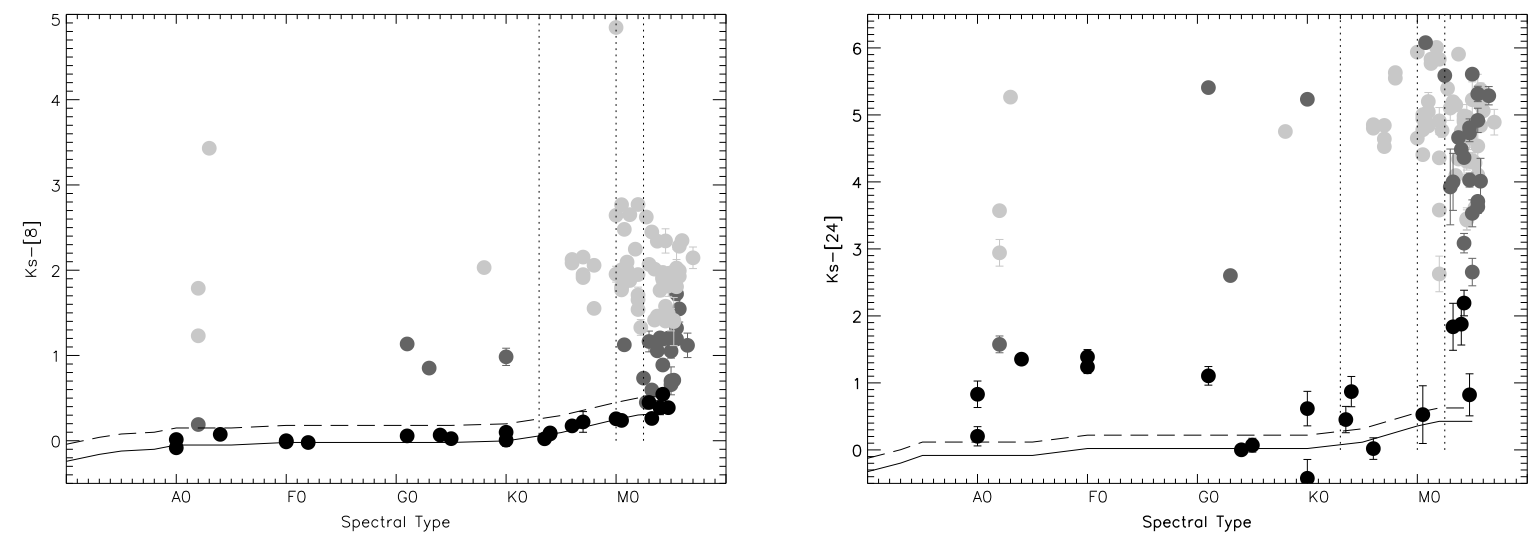

Fig. 12.- $\mathrm{K}_{s^{-}}[8]$ (left panel) and $\mathrm{K}_{s^{-}}[24]$ (right panel) colors vs. spectral type for sources with MIPS $24 \mu \mathrm{m}$ detections. Sources are divided by their IRAC slope: 'thick' sources are light grey dots, 'anemic' sources are dark grey dots, and 'diskless' sources are black dots. The vertical dotted line divides the sample into stars with probable masses $>1.4 M_{\odot}, 0.8-1.4 \mathrm{M}_{\odot}$, and $<0.8 \mathrm{M}_{\odot}$. The horizontal solid line shows the locus of photospheric colors according to the Kurucz-Lejeune stellar atmosphere models. The long-dashed horizontal line identifies sources with a $>20 \%$ IR excess. 


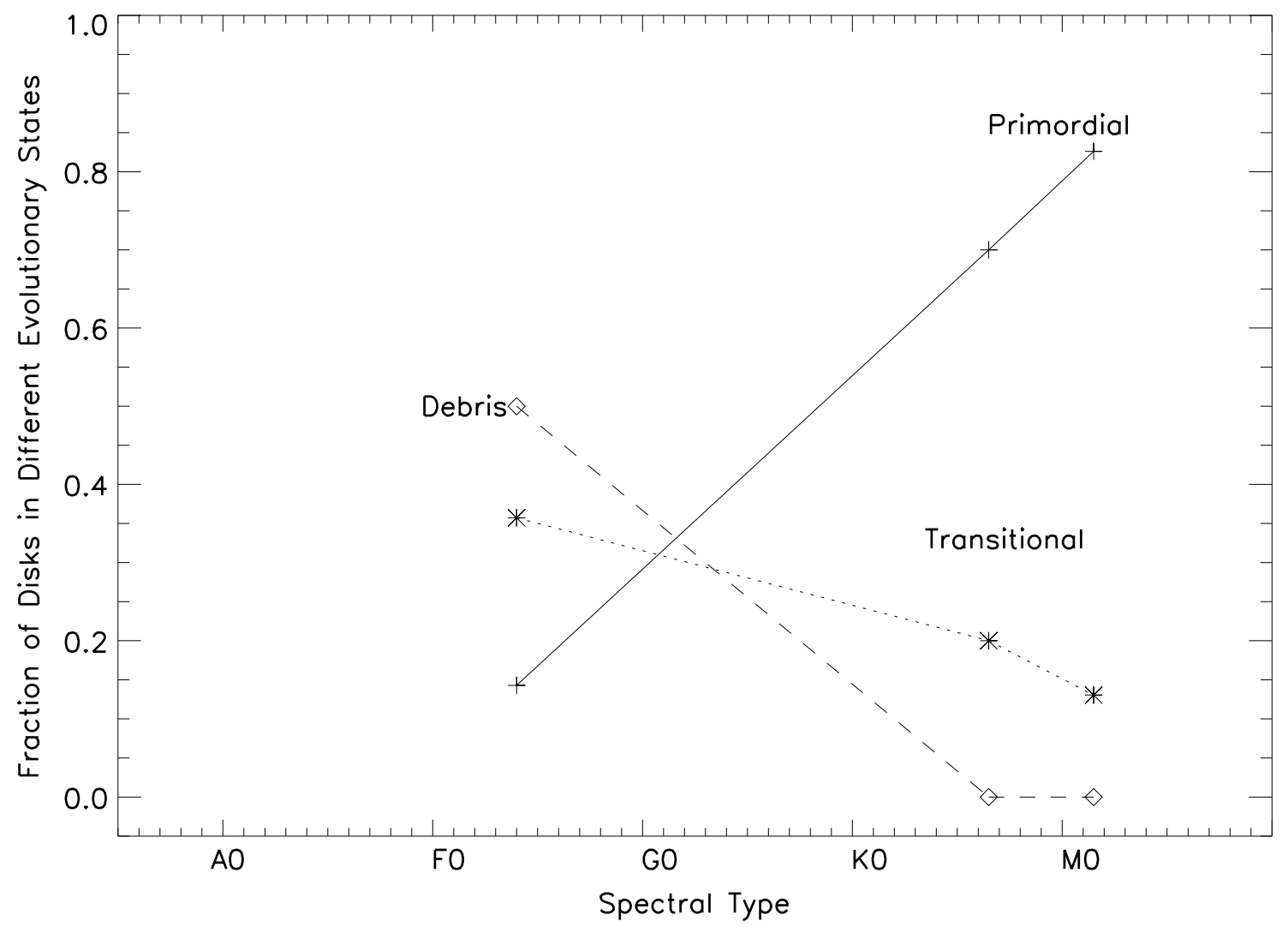

Fig. 13.- Disk evolutionary state from mid-IR colors as a function of stellar mass. The frequencies are given for three stellar mass bins (from left to right): MIPS-detected stars with probable masses $>1.4 \mathrm{M}_{\odot}, 0.8-1.4 \mathrm{M}_{\odot}$, and $0.5-0.8 \mathrm{M}_{\odot}$. 
A. Atlas of SEDs for IC 348 Stars with New MIPS $24 \mu m$ Detections 

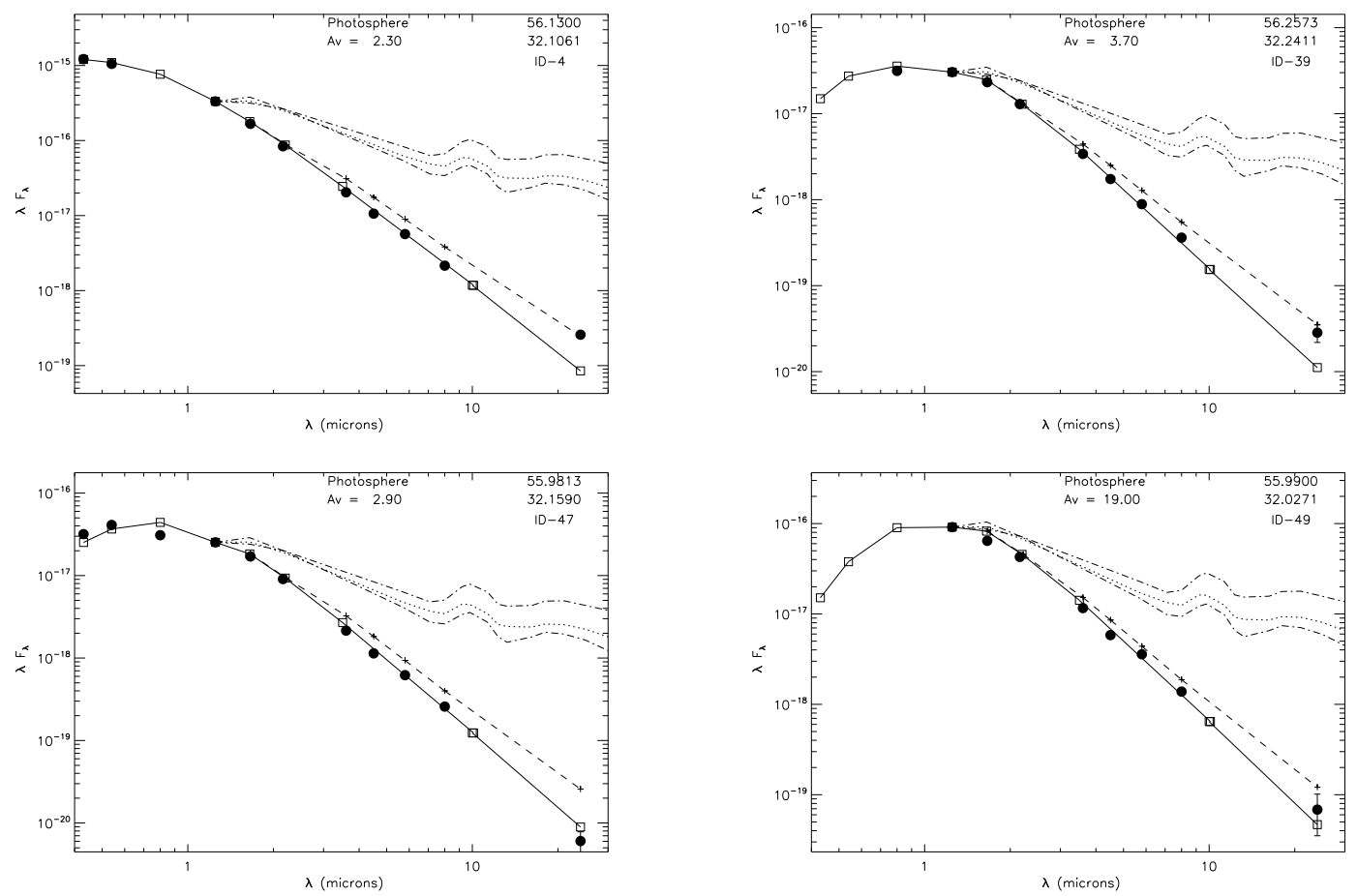

Fig. 14.- Atlas of spectral energy distributions of IC 348 stars with new MIPS $24 \mu \mathrm{m}$ detections. Overplotted are the median Taurus SED (dotted line) with upper and lower quartiles (dot-dashed line). A terrestrial zone debris disk model is shown as a dashed line with mid-IR flux slightly greater than the photosphere (solid line, connected by open squares). 

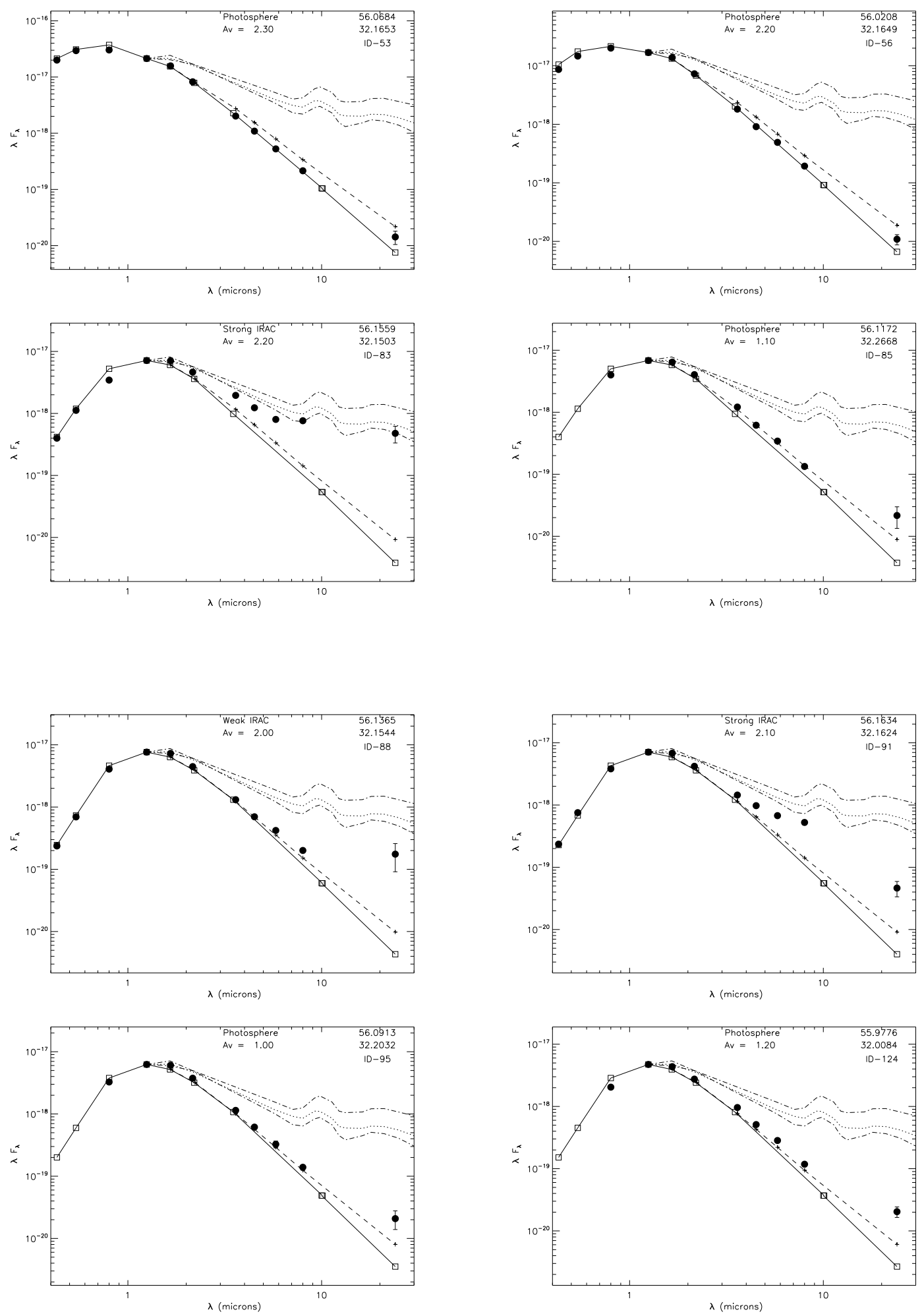

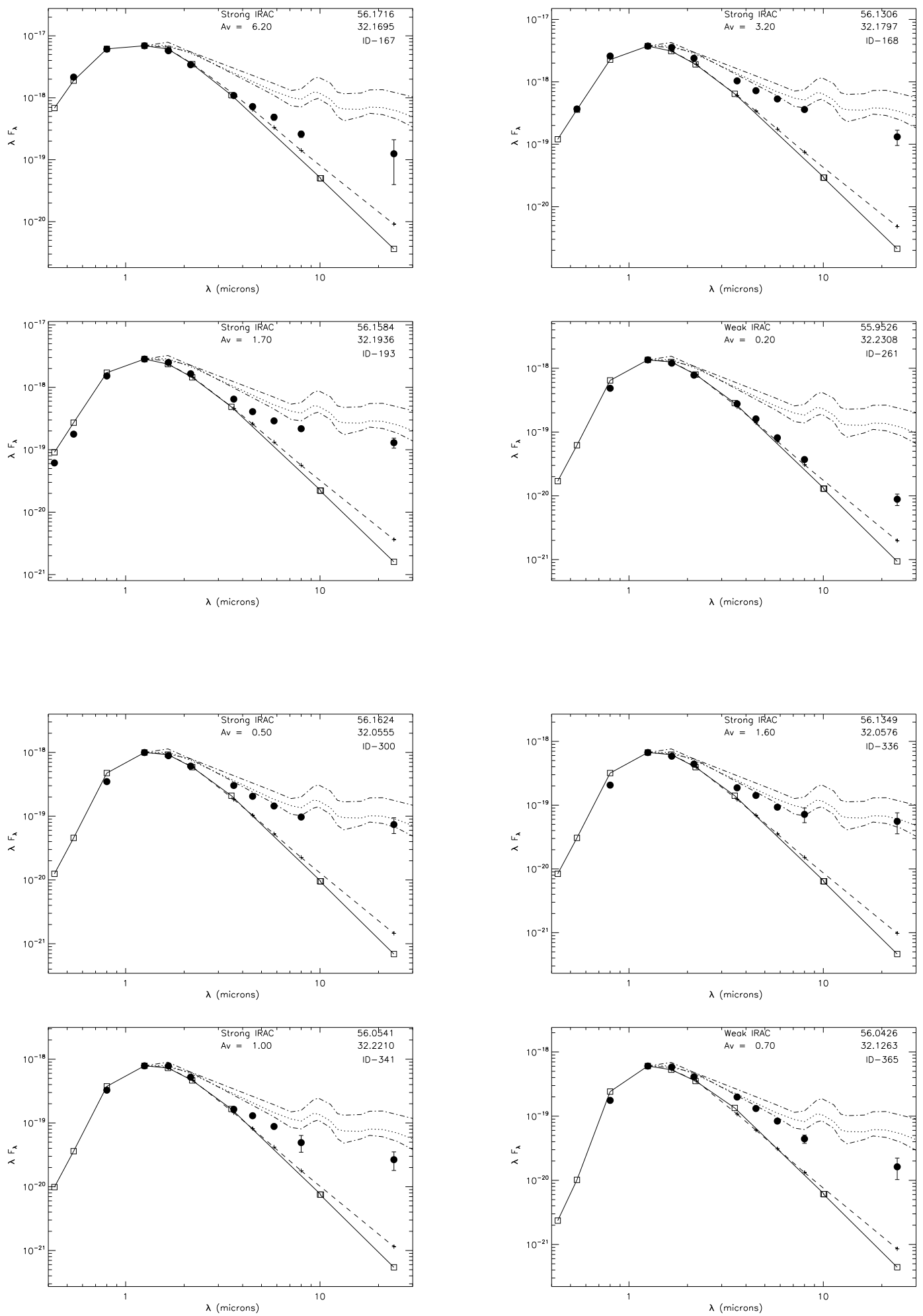

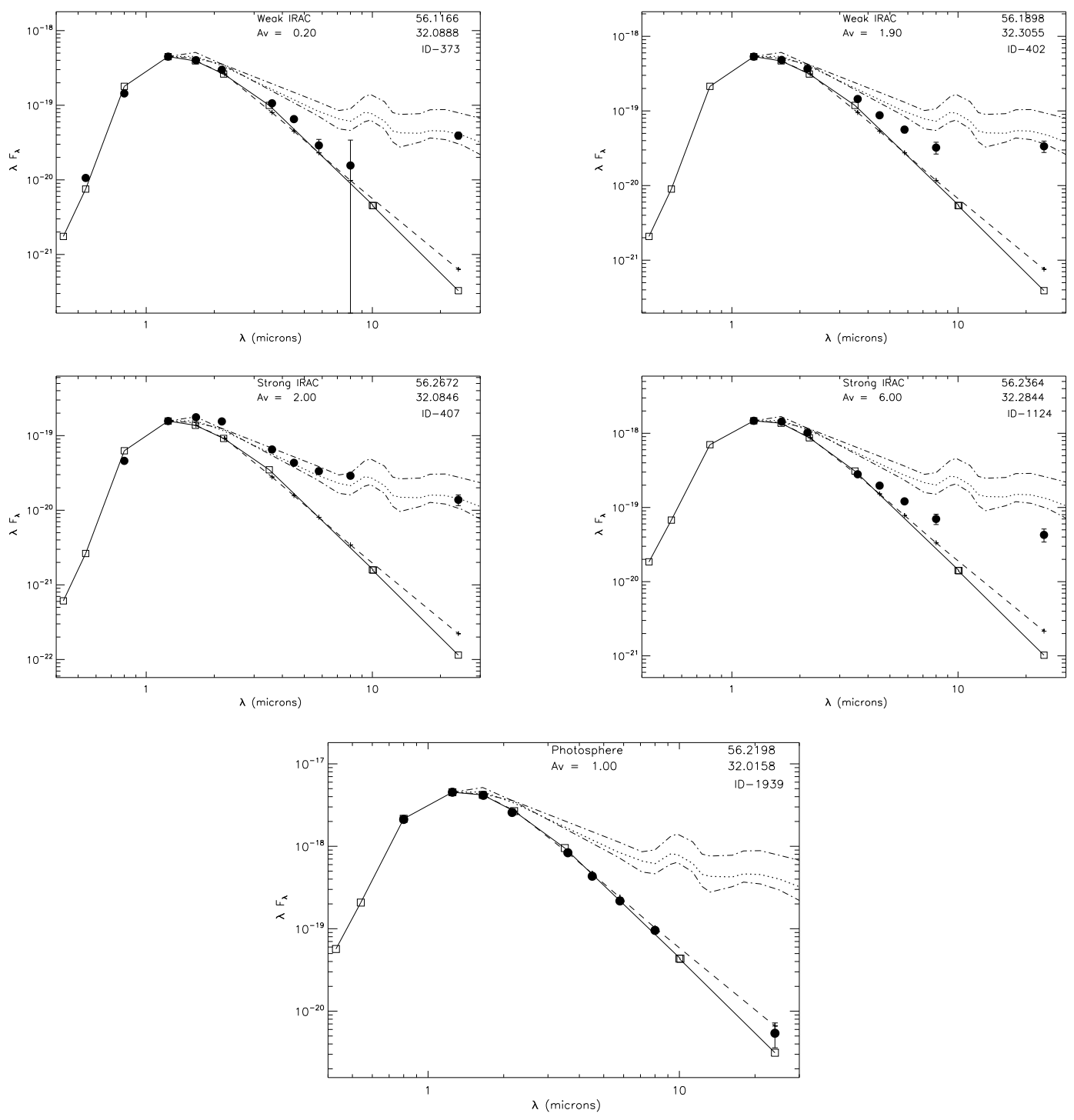